\title{
Topological degree theory and Caputo-Hadamard fractional boundary value problems
}

\author{
Abdelkader Amara', Sina Etemad² and Shahram Rezapour ${ }^{3,4,5^{*}}$ (D)
}

\author{
"Correspondence: \\ shahramrezapour@duytan.edu.vn; \\ sh.rezapour@mail.cmuh.org.tw; \\ sh.rezapour@azaruniv.ac.ir; \\ rezapourshahram@yahoo.ca \\ ${ }^{3}$ Institute of Research and \\ Development, Duy Tan University, \\ Da Nang 550000, Vietnam \\ ${ }^{4}$ Faculty of Natural Sciences, Duy \\ Tan University, Da Nang 550000, \\ Vietnam \\ Full list of author information is \\ available at the end of the article
}

\begin{abstract}
We study two hybrid and non-hybrid fractional boundary value problems via the Caputo-Hadamard type derivatives. We seek the existence criteria for these two problems separately. By utilizing the generalized Dhage's theorem, we derive desired results for an integral structure of solutions for the hybrid problems. Also by considering the special case as a non-hybrid boundary value problem (BVP), we establish other results based on the existing tools in the topological degree theory. In the end of the article, we examine our theoretical results by presenting some numerical examples to show the applicability of the analytical findings.
\end{abstract}

MSC: Primary 34A08; secondary 34A12

Keywords: Caputo-Hadamard fractional BVP; Condensing operator; Degree theory; The generalized Dhage's theorem

\section{Introduction}

The fractional calculus has always been one of the most widely used branches of mathematics in other applied and computational sciences. This degree of importance is due to the high flexibility of the tools and operators defined in this theory. On this basis, researchers have been using various powerful fractional operators in recent decades to model different types of existing natural processes in the world. In the meantime, because modeling based on fractional operators yields more accurate numerical results than modeling based on integer order operators, different generalizations of these fractional operators have been introduced by numerous mathematicians.

The fractional operators have developed over the years, and their importance has become apparent more and more to researchers today. Instances of the application of such fractional operators can be found in various sciences such as biomathematics, electrical circuits, medicine, etc. [1-15]. All of these reasons have led researchers to find many aspects of the structure of the fractional boundary value problems and the hereditary properties of their solutions. In this regard, many researchers have been investigating advanced fractional models [16-18] and related theoretical results and qualitative behaviors of such boundary value problems [19-28].

(c) The Author(s) 2020. This article is licensed under a Creative Commons Attribution 4.0 International License, which permits use, sharing, adaptation, distribution and reproduction in any medium or format, as long as you give appropriate credit to the original author(s) and the source, provide a link to the Creative Commons licence, and indicate if changes were made. The images or other third party material in this article are included in the article's Creative Commons licence, unless indicated otherwise in a credit line to the material. If material is not included in the article's Creative Commons licence and your intended use is not permitted by statutory regulation or exceeds the permitted use, you will need to obtain permission directly from the copyright holder. To view a copy of this licence, visit http://creativecommons.org/licenses/by/4.0/. 
The fractional operators utilized in models of the current paper are the Hadamard and Caputo-Hadamard integration and differentiation operators, respectively. In this regard, one can point to some papers based on these operators; see, for example, [29-32]. In more recent decades, the attention of researchers has been focused on designing newer fractional hybrid BVPs subject to hybrid or non-hybrid conditions. For more details, see [33-39]. More precisely, this novel aspect of fractional modeling initiated with a research manuscript proposed by Dhage and Lakshmikantham in 2010 (see [40]). They turned to a new family of differential equation entitled hybrid differential equation and then established some useful existence criteria of extremal solutions by utilizing some basic inequalities [40]. Two years later, Zhao et al. extended their work to fractional type models and formulated a BVP relying on fractional hybrid differential equations [41]. Later, Ullah et al. continued this process and employed a new structure of hybrid fractional modeling in which both boundary conditions are presented in the hybrid framework by follows:

$$
\left\{\begin{array}{l}
\mathcal{R} \mathcal{D}_{0^{+}}^{\kappa^{*}}\left[\frac{y(t)-p(t, y(t))}{q(t, y(t))}\right]=\psi(t, y(t)), \quad t \in[0,1], \\
{\left.\left[\frac{y(t)-p(t, y(t))}{q(t, y(t))}\right]\right|_{t=0}=0,\left.\quad\left[\frac{y(t)-p(t, y(t))}{q(t, y(t))}\right]\right|_{t=1}=0,}
\end{array}\right.
$$

where $q \in \mathcal{C}_{\mathbb{R} \neq 0}([0,1] \times \mathbb{R})$ is nonzero, both $p$ and $\psi$ are continuous real-valued functions on $[0,1] \times \mathbb{R}$ and ${ }^{\mathcal{R}} \mathcal{D}_{0^{+}}^{\kappa^{*}}$ represents the Riemann-Liouville derivative of order $\kappa^{*} \in(0,1]$ [42]. In 2020, Baleanu et al. presented a novel construction of a fractional hybrid model of a thermostat in which the thermostat controls the amount of heat based on the temperature detected by its sensors [16]. This hybrid model is described by

$$
{ }^{\mathcal{C}} \mathcal{D}_{0^{+}}^{\kappa^{*}}\left[\frac{y(t)}{q(t, y(t))}\right]+\Phi(t, y(t))=0, \quad \kappa^{*} \in(1,2], t \in[0,1],
$$

with the fractional hybrid boundary conditions

$$
\left\{\begin{array}{l}
\left.\mathcal{D}\left[\frac{y(t)}{q(t, y(t))}\right]\right|_{t=0}=0 \\
\left.\lambda^{\mathcal{C}} \mathcal{D}_{0^{+}}^{\kappa^{*}-1}\left[\frac{y(t)}{q(t, y(t))}\right]\right|_{t=1}+\left.\left[\frac{y(t)}{q(t, y(t))}\right]\right|_{t=\eta}=0
\end{array}\right.
$$

where $\lambda>0$ denotes an arbitrary parameter, $\eta \in[0,1]$, and $\kappa^{*}-1 \in(0,1]$. Moreover, $\mathcal{D}=$ $\frac{\mathrm{d}}{\mathrm{d} t},{ }^{\mathcal{C}} \mathcal{D}_{0^{+}}^{\gamma}$ is the Caputo derivative of order $\gamma \in\left\{\kappa^{*}, \kappa^{*}-1\right\}, \Phi \in \mathcal{C}_{\mathbb{R}}([0,1] \times \mathbb{R})$, and $q \in$ $\mathcal{C}_{\mathbb{R}^{70}}([0,1] \times \mathbb{R})$ is nonzero [16]. By using the main ideas of these works, we are going to investigate the Caputo-Hadamard fractional hybrid differential equation

$$
{ }^{\mathcal{C H}} \mathcal{D}_{1^{+}}^{\kappa^{*}}\left[\frac{y(t)-\Lambda\left(t, y(t), \lambda_{1}^{*} \int_{1}^{e} y(\varpi) \mathrm{d} \varpi,{ }^{\mathcal{H}} \mathcal{I}_{1^{+}}^{\gamma^{*}} y(t)\right)}{\Psi\left(t, y(t), \lambda_{2}^{*} \int_{1}^{e} y(\varpi) \mathrm{d} \varpi,{ }^{\mathcal{H}} \mathcal{I}_{1^{+}}^{\mu^{*}} y(t)\right)}\right]=\hat{\Upsilon}(t, y(t)), \quad t \in[1, e],
$$

with the mixed Hadamard integral hybrid boundary value conditions

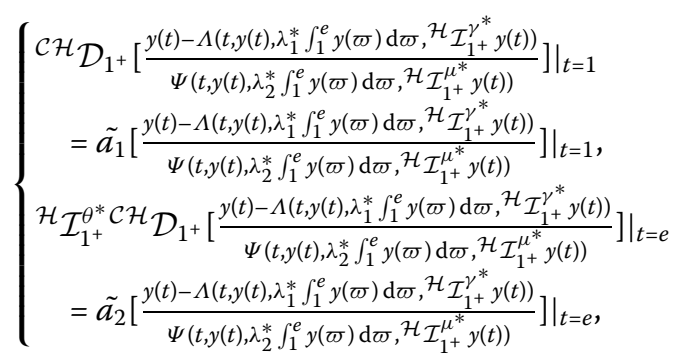


where $\kappa^{*} \in(1,2], \gamma^{*}, \mu^{*}, \theta^{*}>0$, and $\lambda_{1}^{*}, \lambda_{2}^{*}, \tilde{a_{1}}, \tilde{a_{2}} \in \mathbb{R}$. Here, ${ }^{\mathcal{C H}} \mathcal{D}_{1^{+}}^{\kappa^{*}}$ represents the CaputoHadamard fractional derivative of order $\kappa^{*},{ }^{\mathcal{H}} \mathcal{I}_{1^{+}}^{\eta}$ is the Hadamard fractional integral of order $\eta \in\left\{\gamma^{*}, \mu^{*}, \theta^{*}\right\}, \Psi:[1, e] \times \mathbb{R}^{3} \rightarrow \mathbb{R} \backslash\{0\}$ is a nonzero continuous map, $\Lambda \in$ $\mathcal{C}_{\mathbb{R}}\left([1, e] \times \mathbb{R}^{3}\right)$, and $\hat{\Upsilon} \in \mathcal{C}_{\mathbb{R}}([1, e] \times \mathbb{R})$.

By reviewing other papers published in recent years, we find that some researchers have combined the existence theory with the topological degree theory and studied different models using the existing analytical notions in this theory. For instance, one can point to some published papers in this regard, such as [43-48]. In the light of this, we address a special case of the Caputo-Hadamard hybrid BVP (1)-(2) in the sequel of the present paper. In other words, we set $\Lambda\left(t, y(t), \lambda_{1}^{*} \int_{1}^{e} y(\varpi) \mathrm{d} \varpi,{ }^{\mathcal{H}} \mathcal{I}_{1^{+}}^{\gamma^{*}} y(t)\right)=0$ and $\Psi\left(t, y(t), \lambda_{2}^{*} \int_{1}^{e} y(\varpi) \mathrm{d} \varpi,{ }^{\mathcal{H}} \mathcal{I}_{1^{+}}^{\mu^{*}} y(t)\right)=1$. Then the Caputo-Hadamard fractional hybrid BVP (1)-(2) reduces to the following Caputo-Hadamard fractional non-hybrid BVP:

$$
\left\{\begin{array}{l}
\mathcal{C H}^{\mathcal{D}_{1^{+}}} \kappa^{*} y(t)=\hat{\Upsilon}(t, y(t)), \\
\mathcal{C H}^{\mathcal{H}} \mathcal{D}_{1^{+}} y(1)=\tilde{a_{1}} y(1), \quad \frac{1}{\Gamma\left(\theta^{*}\right)} \int_{1}^{e}\left(\ln \frac{e}{\varpi}\right)^{\theta^{*}-1}\left[{ }^{\mathcal{C H}} \mathcal{D}_{1^{+}} y(\varpi)\right] \frac{\mathrm{d} \sigma}{\varpi}=\tilde{a_{2}} y(e) .
\end{array}\right.
$$

For this non-hybrid BVP, we will apply a new approach based on the topological degree theory. Note that both hybrid and non-hybrid BVPs (1)-(2) and (3) are novel in the sense that boundary conditions are written as mixed Hadamard integral and Caputo-Hadamard derivative simultaneously.

\section{Preliminaries}

First, some important and necessary preliminaries on the fractional calculus are recalled in this section. Assume that $\kappa^{*} \geq 0$. The Hadamard fractional integral of $y \in \mathcal{C}_{\mathbb{R}}([a, b])$ of order $\kappa^{*}$ is given by ${ }^{\mathcal{H}} \mathcal{I}_{a^{+}}^{0}(y(t))=y(t)$ and ${ }^{\mathcal{H}} \mathcal{I}_{a^{+}}^{\kappa^{*}}(y(t))=\frac{1}{\Gamma\left(\kappa^{*}\right)} \int_{a}^{t}\left(\ln \frac{t}{\sigma}\right)^{\left(\kappa^{*}-1\right)} y(\varpi) \frac{\mathrm{d} \sigma}{\varpi}$ whenever the RHS-integral has finite value $[49,50]$. Note that for each $\kappa_{1}^{*}, \kappa_{2}^{*} \in \mathbb{R}^{+}$, we have $\left.\mathcal{H}_{\mathcal{I}_{a^{+}}}^{\kappa_{1}^{*}} \mathcal{H}^{\mathcal{I}_{a^{+}}^{\kappa_{2}^{*}}} y(t)\right)={ }^{\mathcal{H}} \mathcal{I}_{a^{+}}^{\kappa_{1}^{*}+\kappa_{2}^{*}} y(t)$ and ${ }^{\mathcal{H}} \mathcal{I}_{a^{+}}^{\kappa_{1}^{*}}\left(\ln \frac{t}{a}\right)^{\kappa_{2}^{*}}=\frac{\Gamma\left(\kappa_{2}^{*}+1\right)}{\Gamma\left(\kappa_{1}^{*}+\kappa_{2}^{*}+1\right)}\left(\ln \frac{t}{a}\right)^{\kappa_{1}^{*}+\kappa_{2}^{*}}$ for $t>a$ [50]. It is obvious that $\mathcal{H}_{\mathcal{I}_{a^{+}}^{\kappa_{1}^{*}}} 1=\frac{1}{\Gamma\left(\kappa_{1}^{*}+1\right)}\left(\ln \frac{t}{a}\right)^{\kappa_{1}^{*}}$ for any $t>a$ by setting $\kappa_{2}^{*}=0$ [50]. Now, let $n=\left[\kappa^{*}\right]+1$. The Hadamard fractional derivative of order $\kappa^{*}$ for a function $y:(a, b) \rightarrow \mathbb{R}$ is introduced by ${ }^{\mathcal{H}} \mathcal{D}_{a^{+}}^{\kappa^{*}}(y(t))=\frac{1}{\Gamma\left(n-\kappa^{*}\right)}\left(t \frac{\mathrm{d} t}{t}\right)^{n} \int_{a}^{t}\left(\ln \frac{t}{\varpi}\right)^{\left(n-\kappa^{*}-1\right)} y(\varpi) \frac{\mathrm{d} \omega}{\varpi}$ provided that the RHSintegral has finite value $[49,50]$. The Caputo-Hadamard fractional derivative of order $\kappa^{*}$ for $y \in \mathcal{A C}_{\mathbb{R}}^{n}([a, b])$ is represented by

$$
{ }^{\mathcal{H}} \mathcal{D}_{a^{+}}^{\kappa^{*}}(y(t))=\frac{1}{\Gamma\left(n-\kappa^{*}\right)} \int_{a}^{t}\left(\ln \frac{t}{\varpi}\right)^{\left(n-\kappa^{*}-1\right)}\left(t \frac{\mathrm{d} t}{t}\right)^{n} y(\varpi) \frac{\mathrm{d} \varpi}{\varpi}
$$

whenever the RHS-integral has finite value $[49,50]$. Now assume that $y \in \mathcal{A C}_{\mathbb{R}}^{n}([a, b])$ and $n-1<\kappa^{*} \leq n$. In the monograph [50], it is verified that the general solution of the Caputo-Hadamard differential equation ${ }^{\mathcal{C H}} \mathcal{D}_{a^{+}}^{\kappa^{*}}(y(t))=0$ is obtained of the form $y(t)=$ $\sum_{j=0}^{n-1} m_{j}^{*}\left(\ln \frac{t}{a}\right)^{j}$, and so we have

$$
{ }^{\mathcal{H}} \mathcal{I}_{a^{+}}^{\kappa^{*}}\left({ }^{\mathcal{H}} \mathcal{D}_{a^{+}}^{\kappa^{*}} y(t)\right)=y(t)+m_{0}^{*}+m_{1}^{*}\left(\ln \frac{t}{a}\right)+m_{2}^{*}\left(\ln \frac{t}{a}\right)^{2}+\cdots+m_{n-1}^{*}\left(\ln \frac{t}{a}\right)^{n-1}
$$

for any $t>a$. In the following, we review some notions and results on the topological degree theory which are useful throughout the paper. Let $\mathbb{B}$ represent the collection of all 
bounded sets in a Banach space $\mathcal{X}$. The Kuratowski's measure of noncompactness $\mu: \mathbb{B} \rightarrow$ $\mathbb{R}^{+}$is defined by $\mu(\mathcal{B}):=\inf \left\{\epsilon>0: \mathcal{B}=\bigcup_{j=1}^{n} \mathcal{B}_{j}\right.$ and $\operatorname{diam}\left(\mathcal{B}_{i}\right) \leq \epsilon$ for $\left.j=1, \ldots, n\right\}$, where $\operatorname{diam}\left(\mathcal{B}_{j}\right)=\sup \left\{\left|y-y^{\prime}\right|: y, y^{\prime} \in \mathcal{B}_{j}\right\}$ and $\mathcal{B}$ is a bounded element of $\mathbb{B}$. It is evident that $0 \leq$ $\mu(\mathcal{B}) \leq \operatorname{diam}(\mathcal{B})<+\infty[51,52]$.

Lemma $1([51,52])$ Let $\mathcal{X}$ be an arbitrary real Banach space and $\mathcal{B}, \mathcal{E} \in \mathbb{B}$ be bounded subsets of $\mathcal{X}$. Then the following statements are valid:

(a1) $\mathcal{B}$ is relatively compact if and only if $\mu(\mathcal{B})=0$;

(a2) $\mu(\mathcal{B})=\mu(\overline{\mathcal{B}})=\mu(\operatorname{cnvx}(\mathcal{B}))$, where $\overline{\mathcal{B}}$ and $\operatorname{cnvx}(\mathcal{B})$ represent the closure and convex hull of $\mathcal{B}$, respectively;

(a3) If $\mathcal{B} \subseteq \mathcal{E}$, then $\mu(\mathcal{B}) \leq \mu(\mathcal{E})$;

(a4) $\mu(\lambda+\mathcal{E}) \leq \mu(\mathcal{E})$ for each $\lambda \in \mathbb{R}$;

(a5) $\mu(\lambda \mathcal{B})=|\lambda| \mu(\mathcal{B})$ for each $\lambda \in \mathbb{R}$;

(a6) $\mu(\mathcal{B}+\mathcal{E}) \leq \mu(\mathcal{B})+\mu(\mathcal{E})$ so that $\mathcal{B}+\mathcal{E}=\left\{y+y^{\prime} ; y \in \mathcal{B}, y^{\prime} \in \mathcal{E}\right\}$;

(a7) $\mu(\mathcal{B} \cup \mathcal{E}) \leq \max \{\mu(\mathcal{B}), \mu(\mathcal{E})\}$.

Note that conditions (a5) and (a6) mean that $\mu$ is a seminorm. Let $\mathcal{B} \in \mathbb{B}$ be a bounded subset of a Banach space $\mathcal{X}$. We say that a continuous bounded map $\Phi: \mathcal{B} \rightarrow \mathcal{X}$ is $\mu$ Lipschitz if there is a constant $\tilde{K}^{*} \geq 0$ such that $\mu(\Phi(\mathcal{B})) \leq \tilde{K}^{*} \mu(\mathcal{B})$. Also, $\Phi$ is called a strict $\mu$-contraction if $\tilde{K}^{*}$ is less than one [51]. A $\mu$-condensing function $\Phi$ is supposed to satisfy $\mu(\Phi(\mathcal{B})) \leq \mu(\mathcal{B})$ for each $\mathcal{B} \in \mathbb{B}$ with $\mu(\mathcal{B})>0$. Indeed, the inequality $\mu(\Phi(\mathcal{B})) \geq$ $\mu(\mathcal{B})$ implies that $\mu(\mathcal{B})=0[51]$.

Proposition 2 ([53]) Let $\Phi: \mathcal{B} \rightarrow \mathcal{X}$ be Lipschitz with constant $\tilde{K}^{*}$ where $\mathcal{B} \subset \mathcal{X}$. Then $\Phi$ is $\mu$-Lipschitz with the same constant $\tilde{K}^{*}$.

Proposition 3 ([53]) For every $\mathcal{B} \subset \mathcal{X}$, if $\Phi: \mathcal{B} \rightarrow \mathcal{X}$ is compact, then $\Phi$ is $\mu$-Lipschitz with constant $\tilde{K}^{*}=0$.

Proposition 4 ([53]) For each $\mathcal{B} \subset \mathcal{X}$, assume that $\Phi_{1}, \Phi_{2}: \mathcal{B} \rightarrow \mathcal{X}$ are two $\mu$-Lipschitz operators with constant $\tilde{K}_{1}^{*}$ and $\tilde{K}_{2}^{*}$, respectively. Then $\Phi_{1}+\Phi_{2}: \mathcal{B} \rightarrow \mathcal{X}$ is $\mu$-Lipschitz with constant $\tilde{K}_{1}^{*}+\tilde{K}_{2}^{*}$.

The following theorem due to Dhage is utilized for our result related to the mixed Caputo-Hadamard hybrid BVP (1)-(2).

Theorem 5 ([54]) Let $\mathcal{X}$ be a Banach algebra and $\mathcal{B}$ be a convex bounded closed nonempty subset of $\mathcal{X}$. Moreover, suppose that three operators $\Phi_{1}, \Phi_{2}: \mathcal{X} \rightarrow \mathcal{X}$ and $\Phi_{3}: \mathcal{B} \rightarrow \mathcal{X}$ satisfy the following three assumptions:

(i) $\Phi_{1}$ and $\Phi_{2}$ are Lipschitz with constants $\tilde{K}_{1}^{*}$ and $\tilde{K}_{2}^{*}$, respectively,

(ii) $\Phi_{3}$ is compact and continuous,

(iii) $\tilde{K}_{1}^{*} \hat{\Delta}+\tilde{K}_{2}^{*}<1$ so that $\hat{\Delta}=\left\|\Phi_{3}(\mathcal{B})\right\|_{\mathcal{X}}=\sup \left\{\left\|\Phi_{3} y\right\|_{\mathcal{X}}: y \in \mathcal{B}\right\}$.

Then either $(a)$ the equation $\left(\Phi_{1} y\right)\left(\Phi_{3} y\right)+\left(\Phi_{2} y\right)=y$ has a solution belonging to $\mathcal{B}$ or $(b)$ for each $r>0$, there is $v^{*} \in \mathcal{X}$ with $\left\|v^{*}\right\|_{\mathcal{X}}=$ r provided that $\alpha_{0}\left(\Phi_{1} v^{*}\right)\left(\Phi_{3} v^{*}\right)+\alpha_{0}\left(\Phi_{2} v^{*}\right)=v^{*}$ for some $\alpha_{0} \in(0,1)$.

The following theorem due to Isaia is utilized for our result related to the mixed CaputoHadamard non-hybrid BVP (3). 
Theorem 6 ([53]) Let $\Phi: \mathcal{X} \rightarrow \mathcal{X}$ be a $\mu$-condensing operator on the Banach space $\mathcal{X}$ and assume that

$$
\mathcal{B}=\{y \in \mathcal{X} \text { : there is } \lambda \in[0,1] \text { such that } y=\lambda(\Phi y)\} .
$$

If $\mathcal{B}$ is a bounded set in $\mathcal{X}$, so that there is a number $\rho>0$ such that $\mathcal{B} \subset \overline{\mathcal{V}_{\rho}(0)}$, then we have $\operatorname{deg}\left(I-\lambda \Phi, \overline{\mathcal{V}_{\rho}(0)}, 0\right)=1$. Moreover, $\Phi$ has at least one fixed point, and the family of all fixed points of $\Phi$ belongs to $\overline{\mathcal{V}_{\rho}(0)}$.

\section{Main results}

Now, we are ready to derive the desired analytical findings. For this reason, we build a new space as $\mathcal{X}=\left\{y(t): y(t) \in \mathcal{C}_{\mathbb{R}}([1, e])\right\}$ supplemented with the sup-norm $\|y\|_{\mathcal{X}}=$ $\sup _{t \in[1, e]}|y(t)|$ and the multiplication action on $\mathcal{X}$ by $\left(y \cdot y^{\prime}\right)(t)=y(t) y^{\prime}(t)$ for each $y, y^{\prime} \in \mathcal{X}$. Then it is easily verified that an ordered triple $\left(\mathcal{X},\|\cdot\|_{\mathcal{X}}, \cdot\right)$ is a Banach algebra. In the following lemma, we derive an integral structure for the solution of the hybrid BVP (1)(2).

Lemma 7 Let $g \in \mathcal{X}$. Then a function $\tilde{y_{0}}$ is a solution for the Caputo-Hadamard hybrid equation

$$
{ }^{\mathcal{C H}} \mathcal{D}_{1^{+}}^{\kappa^{*}}\left[\frac{y(t)-\Lambda\left(t, y(t), \lambda_{1}^{*} \int_{1}^{e} y(\varpi) \mathrm{d} \varpi,{ }^{\mathcal{H}} \mathcal{I}_{1^{+}}^{\gamma^{*}} y(t)\right)}{\Psi\left(t, y(t), \lambda_{2}^{*} \int_{1}^{e} y(\varpi) \mathrm{d} \varpi,{ }^{\mathcal{H}} \mathcal{I}_{1^{+}}^{\mu^{*}} y(t)\right)}\right]=g(t)
$$

furnished with mixed Hadamard integral hybrid boundary value conditions

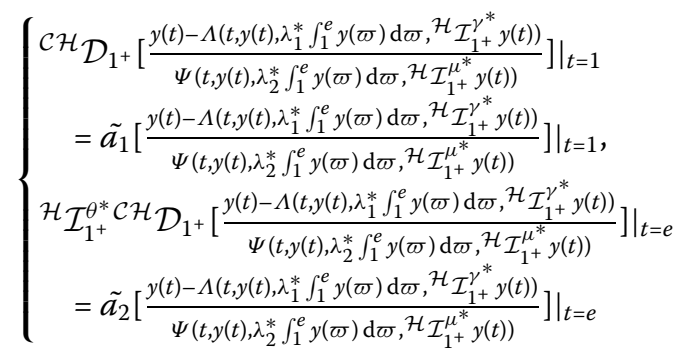

if and only if $\tilde{y}_{0}$ is a solution for the Hadamard integral equation

$$
\begin{aligned}
y(t)= & \Psi\left(t, y(t), \lambda_{2}^{*} \int_{1}^{e} y(\varpi) \mathrm{d} \varpi,{ }^{\mathcal{H}} \mathcal{I}_{1^{+}}^{\mu^{*}} y(t)\right) \\
& \times\left(\frac{1}{\Gamma\left(\kappa^{*}\right)} \int_{1}^{t}\left(\ln \frac{t}{\varpi}\right)^{\kappa^{*}-1} g(\varpi) \frac{\mathrm{d} \varpi}{\varpi}\right. \\
& +\frac{\tilde{a_{2}}\left(1+\tilde{a_{1}} \ln (t)\right)}{\mathcal{Q}^{*} \Gamma\left(\kappa^{*}\right)} \int_{1}^{e}\left(\ln \frac{e}{\varpi}\right)^{\kappa^{*}-1} g(\varpi) \frac{\mathrm{d} \varpi}{\varpi} \\
& \left.-\frac{\left(1+\tilde{a_{1}} \ln (t)\right)}{\mathcal{Q}^{*} \Gamma\left(\kappa^{*}+\theta^{*}-1\right)} \int_{1}^{e}\left(\ln \frac{e}{\varpi}\right)^{\kappa^{*}+\theta^{*}-2} g(\varpi) \frac{\mathrm{d} \varpi}{\varpi}\right) \\
& +\Lambda\left(t, y(t), \lambda_{1}^{*} \int_{1}^{e} y(\varpi) \mathrm{d} \varpi,{ }^{\mathcal{H}} \mathcal{I}_{1^{+}}^{\gamma^{*}} y(t)\right),
\end{aligned}
$$


where

$$
\mathcal{Q}^{*}=\left|\frac{\tilde{a_{1}}-\left(1+\tilde{a_{1}}\right) \tilde{a_{2}} \Gamma\left(\theta^{*}+1\right)}{\Gamma\left(\theta^{*}+1\right)}\right| \neq 0 .
$$

Proof As a first step, we assume that $\tilde{y_{0}}$ is a solution for the hybrid differential equation (4). Then, by properties of the $\kappa^{*}$ th order Hadamard integral, we seek constants $m_{0}^{*}, m_{1}^{*} \in \mathbb{R}$ such that

$$
\begin{aligned}
& \frac{\tilde{y_{0}}(t)-\Lambda\left(t, \tilde{y_{0}}(t), \lambda_{1}^{*} \int_{1}^{e} \tilde{y_{0}}(\varpi) \mathrm{d} \varpi, \mathcal{H}_{1_{1}}^{\gamma^{*}} \tilde{y}_{0}(t)\right)}{\Psi\left(t, \tilde{y_{0}}(t), \lambda_{2}^{*} \int_{1}^{e} \tilde{y_{0}}(\varpi) \mathrm{d} \varpi, \mathcal{H}_{1^{+}}^{\mu^{*}} \tilde{y_{0}}(t)\right)} \\
& \quad=\frac{1}{\Gamma\left(\kappa^{*}\right)} \int_{1}^{t}\left(\ln \frac{t}{\varpi}\right)^{\kappa^{*}-1} g(\varpi) \frac{\mathrm{d} \varpi}{\varpi}+m_{0}^{*}+m_{1}^{*}(\ln t)
\end{aligned}
$$

and so

$$
\begin{aligned}
\tilde{y_{0}}(t)= & \Lambda\left(t, \tilde{y_{0}}(t), \lambda_{1}^{*} \int_{1}^{e} \tilde{y_{0}}(\varpi) \mathrm{d} \varpi,{ }^{\mathcal{H}} \mathcal{I}_{1^{+}}^{\gamma^{*}} \tilde{y}_{0}(t)\right) \\
& +\Psi\left(t, \tilde{y_{0}}(t), \lambda_{2}^{*} \int_{1}^{e} \tilde{y_{0}}(\varpi) \mathrm{d} \varpi,{ }^{\mathcal{H}} \mathcal{I}_{1^{+}}^{\mu^{*}} \tilde{y_{0}}(t)\right) \\
& \times\left(\frac{1}{\Gamma\left(\kappa^{*}\right)} \int_{1}^{t}\left(\ln \frac{t}{\varpi}\right)^{\kappa^{*}-1} g(\varpi) \frac{\mathrm{d} \varpi}{\varpi}+m_{0}^{*}+m_{1}^{*}(\ln t)\right) .
\end{aligned}
$$

Thus,

$$
\begin{aligned}
& { }^{\mathcal{H}} \mathcal{D}_{1^{+}}\left[\frac{\tilde{y_{0}}(t)-\Lambda\left(t, \tilde{y_{0}}(t), \lambda_{1}^{*} \int_{1}^{e} \tilde{y_{0}}(\varpi) \mathrm{d} \varpi,{ }^{\mathcal{H}} \mathcal{I}_{1^{+}}^{\gamma^{*}} \tilde{y_{0}}(t)\right)}{\Psi\left(t, \tilde{y_{0}}(t), \lambda_{2}^{*} \int_{1}^{e} \tilde{y_{0}}(\varpi) \mathrm{d} \varpi,{ }^{\mathcal{H}} \mathcal{I}_{1^{+}}^{\mu^{*}} \tilde{y_{0}}(t)\right)}\right] \\
& =\frac{1}{\Gamma\left(\kappa^{*}-1\right)} \int_{1}^{t}\left(\ln \frac{t}{\varpi}\right)^{\kappa^{*}-2} g(\varpi) \frac{\mathrm{d} \varpi}{\varpi}+m_{1}^{*}, \\
& { }^{\mathcal{H}} \mathcal{I}_{1^{+}}^{\theta^{*}} \mathcal{C H}_{\mathcal{D}_{1^{+}}}\left[\frac{\tilde{y_{0}}(t)-\Lambda\left(t, \tilde{y_{0}}(t), \lambda_{1}^{*} \int_{1}^{e} \tilde{y_{0}}(\varpi) \mathrm{d} \varpi,{ }^{\mathcal{H}} \mathcal{I}_{1^{+}}^{\gamma^{*}} \tilde{y_{0}}(t)\right)}{\Psi\left(t, \tilde{y_{0}}(t), \lambda_{2}^{*} \int_{1}^{e} \tilde{y_{0}}(\varpi) \mathrm{d} \varpi, \mathcal{H}_{1^{+}}^{\mu^{*}} \tilde{y_{0}}(t)\right)}\right] \\
& =m_{1}^{*} \frac{(\ln t)^{\theta^{*}}}{\Gamma\left(\theta^{*}+1\right)}+\frac{1}{\Gamma\left(\kappa^{*}+\theta^{*}-1\right)} \int_{1}^{t}\left(\ln \frac{t}{\varpi}\right)^{\kappa^{*}+\theta^{*}-2} g(\varpi) \frac{\mathrm{d} \varpi}{\varpi} .
\end{aligned}
$$

In the light of both mixed hybrid boundary conditions given in (5), we obtain

$$
\begin{aligned}
m_{0}^{*}= & \frac{\tilde{a}_{2}}{\mathcal{Q}^{*} \Gamma\left(\kappa^{*}\right)} \int_{1}^{e}\left(\ln \frac{e}{\varpi}\right)^{\kappa^{*}-1} g(\varpi) \frac{\mathrm{d} \varpi}{\varpi} \\
& -\frac{1}{\mathcal{Q}^{*} \Gamma\left(\kappa^{*}+\theta^{*}-1\right)} \int_{1}^{e}\left(\ln \frac{e}{\varpi}\right)^{\kappa^{*}+\theta^{*}-2} g(\varpi) \frac{\mathrm{d} \varpi}{\varpi}
\end{aligned}
$$

and

$$
\begin{aligned}
m_{1}^{*}= & \frac{\tilde{a_{1}} \tilde{a}_{2}}{\mathcal{Q}^{*} \Gamma\left(\kappa^{*}\right)} \int_{1}^{e}\left(\ln \frac{e}{\varpi}\right)^{\kappa^{*}-1} g(\varpi) \frac{\mathrm{d} \varpi}{\varpi} \\
& -\frac{\tilde{a_{1}}}{\mathcal{Q}^{*} \Gamma\left(\kappa^{*}+\theta^{*}-1\right)} \int_{1}^{e}\left(\ln \frac{e}{\varpi}\right)^{\kappa^{*}+\theta^{*}-2} g(\varpi) \frac{\mathrm{d} \varpi}{\varpi} .
\end{aligned}
$$


By inserting the obtained values $m_{0}^{*}$ and $m_{1}^{*}$ into (8), we reach

$$
\begin{aligned}
\tilde{y_{0}}(t)= & \Psi\left(t, \tilde{y_{0}}(t), \lambda_{2}^{*} \int_{1}^{e} \tilde{y_{0}}(\varpi) \mathrm{d} \varpi,{ }^{\mathcal{H}} \mathcal{I}_{1^{+}}^{\mu^{*}} \tilde{y_{0}}(t)\right) \\
& \times\left(\frac{1}{\Gamma\left(\kappa^{*}\right)} \int_{1}^{t}\left(\ln \frac{t}{\varpi}\right)^{\kappa^{*}-1} g(\varpi) \frac{\mathrm{d} \varpi}{\varpi}\right. \\
& +\frac{\tilde{a_{2}}\left(1+\tilde{a_{1}} \ln (t)\right)}{\mathcal{Q}^{*} \Gamma\left(\kappa^{*}\right)} \int_{1}^{e}\left(\ln \frac{e}{\varpi}\right)^{\kappa^{*}-1} g(\varpi) \frac{\mathrm{d} \varpi}{\varpi} \\
& \left.-\frac{\left(1+\tilde{a_{1}} \ln (t)\right)}{\mathcal{Q}^{*} \Gamma\left(\kappa^{*}+\theta^{*}-1\right)} \int_{1}^{e}\left(\ln \frac{e}{\varpi}\right)^{\kappa^{*}+\theta^{*}-2} g(\varpi) \frac{\mathrm{d} \varpi}{\varpi}\right) \\
& +\Lambda\left(t, \tilde{y_{0}}(t), \lambda_{1}^{*} \int_{1}^{e} \tilde{y_{0}}(\varpi) \mathrm{d} \varpi, \mathcal{H}^{\left.\mathcal{I}_{1^{+}}^{\gamma^{*}} \tilde{y_{0}}(t)\right) .}\right.
\end{aligned}
$$

The last equation implies that $\tilde{y}_{0}$ satisfies the Hadamard integral equation (6), and so $\tilde{y}_{0}$ is the solution of the mentioned integral equation. In the opposite direction, we can easily confirm that $\tilde{y_{0}}$ is a solution for the two-point Caputo-Hadamard hybrid BVP (4)-(5) if $\tilde{y}_{0}$ is supposed to be a solution for the Hadamard integral equation (6). This completes the proof.

Now, based on the obtained Hadamard integral equation in the above lemma, we provide an existence criterion for solutions of the mixed Caputo-Hadamard hybrid BVP (1)(2).

Theorem 8 Let $\Psi:[1, e] \times \mathcal{X}^{3} \rightarrow \mathcal{X} \backslash\{0\}$ and $\Lambda:[1, e] \times \mathcal{X}^{3} \rightarrow \mathcal{X}$ and $\hat{\Upsilon}:[1, e] \times \mathcal{X} \rightarrow \mathcal{X}$ be continuous. Moreover, consider the following hypotheses:

$(\mathcal{H P} 1)$ There is a positive bounded mapping $\varrho:[1, e] \rightarrow \mathbb{R}^{+}$so that for each $y_{i}, y_{i}^{\prime} \in \mathcal{X}$,

$$
\left|\Psi\left(t, y_{1}(t), y_{2}(t), y_{3}(t)\right)-\Psi\left(t, y_{1}^{\prime}(t), y_{2}^{\prime}(t), y_{3}^{\prime}(t)\right)\right| \leq \varrho(t) \sum_{1}^{3}\left|y_{i}(t)-y_{i}^{\prime}(t)\right|
$$

$(\mathcal{H P} 2)$ There is a positive bounded mapping $\sigma:[1, e] \rightarrow \mathbb{R}^{+}$such that for each $y_{i}, y_{i}^{\prime} \in \mathcal{X}$,

$$
\left|\Lambda\left(t, y_{1}(t), y_{2}(t), y_{3}(t)\right)-\Lambda\left(t, y_{1}^{\prime}(t), y_{2}^{\prime}(t), y_{3}^{\prime}(t)\right)\right| \leq \sigma(t) \sum_{1}^{3}\left|y_{i}(t)-y_{i}^{\prime}(t)\right|
$$

$(\mathcal{H P} 3)$ There is a positive continuous function $\psi:[1, e] \rightarrow \mathbb{R}^{+}$and a continuous nondecreasing map $\xi:[0, \infty) \rightarrow[0, \infty)$ such that $|\hat{\Upsilon}(t, y(t))| \leq \psi(t) \xi\left(\|y\|_{\mathcal{X}}\right)$ for any $t \in[1, e]$ and $y \in \mathcal{X}$,

$(\mathcal{H P} 4)$ There exists a number $\rho>0$ such that

$$
\begin{aligned}
\rho> & \left(\Psi^{*} \tilde{M} \psi^{*} \xi\left(\|y\|_{\mathcal{X}}\right)+\Lambda^{*}\right) \\
& /\left(1-\varrho^{*}\left[1+\left|\lambda_{2}^{*}(e-1)\right|+\frac{1}{\Gamma\left(\mu^{*}+1\right)}\right] \tilde{M} \psi^{*} \xi(|y| \mathcal{X})\right. \\
& \left.-\sigma^{*}\left[1+\left|\lambda_{1}^{*}(e-1)\right|+\frac{1}{\Gamma\left(\gamma^{*}+1\right)}\right]\right)
\end{aligned}
$$


where we considered $\Psi^{*}=\sup _{t \in[1, e]}|\psi(t, 0,0,0)|, \Lambda^{*}=\sup _{t \in[1, e]}|\Lambda(t, 0,0,0)|, \psi^{*}=$ $\sup _{t \in[1, e]}|\psi(t)|, \varrho^{*}=\sup _{t \in[1, e]}|\varrho(t)|, \sigma^{*}=\sup _{t \in[1, e]}|\sigma(t)|$ and

$$
\tilde{M}=\frac{1}{\Gamma\left(\kappa^{*}+1\right)}+\left|\frac{\tilde{a_{2}}\left(1+\tilde{a_{1}}\right)}{\mathcal{Q}^{*} \Gamma\left(\kappa^{*}+1\right)}\right|+\left|\frac{\left(1+\tilde{a_{1}}\right)}{\mathcal{Q}^{*} \Gamma\left(\kappa^{*}+\theta^{*}\right)}\right|
$$

Then the mixed Caputo-Hadamard hybrid BVP (1)-(2) has at least one solution if

$$
\varrho^{*}\left[1+\left|\lambda_{2}^{*}(e-1)\right|+\frac{1}{\Gamma\left(\mu^{*}+1\right)}\right] \psi^{*} \xi(\|y\| \mathcal{X}) \tilde{M}+\sigma^{*}\left[1+\left|\lambda_{1}^{*}(e-1)\right|+\frac{1}{\Gamma\left(\gamma^{*}+1\right)}\right]<1
$$

Proof For every positive number $\rho \in \mathbb{R}$, we construct the ball $\overline{\mathcal{V}_{\rho}(0)}:=\left\{y(t) \in \mathcal{X}:\|y\|_{\mathcal{X}} \leq\right.$ $\rho$ \} in the Banach algebra $\mathcal{X}$, where $\rho$ satisfies (9). It is well known that $\overline{\mathcal{V}_{\rho}(0)}$ is a convex closed bounded subset of the Banach algebra $\mathcal{X}$. Based on Lemma 7, we introduce three operators $\Phi_{1}, \Phi_{2}: \mathcal{X} \rightarrow \mathcal{X}$ and $\Phi_{3}: \overline{\mathcal{V}_{\rho}(0)} \rightarrow \mathcal{X}$ by

$$
\begin{aligned}
& \left(\Phi_{1} y\right)(t)=\Psi\left(t, y(t), \lambda_{2}^{*} \int_{1}^{e} y(\varpi) \mathrm{d} \varpi,{ }^{\mathcal{H}} \mathcal{I}_{1^{+}}^{\mu^{*}} y(t)\right), \\
& \left(\Phi_{2} y\right)(t)=\Lambda\left(t, y(t), \lambda_{1}^{*} \int_{1}^{e} y(\varpi) \mathrm{d} \varpi,{ }^{\mathcal{H}} \mathcal{I}_{1^{+}}^{\gamma^{*}} y(t)\right)
\end{aligned}
$$

and

$$
\begin{aligned}
\left(\Phi_{3} y\right)(t)= & \frac{1}{\Gamma\left(\kappa^{*}\right)} \int_{1}^{t}\left(\ln \frac{t}{\varpi}\right)^{\kappa^{*}-1} \hat{\Upsilon}(\varpi, y(\varpi)) \frac{\mathrm{d} \varpi}{\varpi} \\
& +\frac{\tilde{a}_{2}\left(1+\tilde{a_{1}} \ln (t)\right)}{\mathcal{Q}^{*} \Gamma\left(\kappa^{*}\right)} \int_{1}^{e}\left(\ln \frac{e}{\varpi}\right)^{\kappa^{*}-1} \hat{\Upsilon}(\varpi, y(\varpi)) \frac{\mathrm{d} \varpi}{\varpi} \\
& -\frac{\left(1+\tilde{a_{1}} \ln (t)\right)}{\mathcal{Q}^{*} \Gamma\left(\kappa^{*}+\theta^{*}-1\right)} \int_{1}^{e}\left(\ln \frac{e}{\varpi}\right)^{\kappa^{*}+\theta^{*}-2} \hat{\Upsilon}(\varpi, y(\varpi)) \frac{\mathrm{d} \varpi}{\varpi}
\end{aligned}
$$

for any $t \in[1, e]$. It is evident that a function $y_{0} \in \mathcal{X}$ is a solution for the mixed Caputo-Hadamard hybrid BVP (1)-(2) whenever $y_{0}$ satisfies the equation $\left(\Phi_{1} y_{0}\right)\left(\Phi_{3} y_{0}\right)+$ $\left(\Phi_{2} y_{0}\right)=y_{0}$. We intend to show that the three operators $\Phi_{1}, \Phi_{2}$ and $\Phi_{3}$ satisfy all conditions of Theorem 5 and thus, by taking into account hypotheses of Theorem 5 , we will find that there exists such a solution function. First of all, we verify that $\Phi_{1}$ is Lipschitz. Let $y_{1}, y_{2} \in \mathcal{X}$. By $(\mathcal{H} \mathcal{P} 1)$, we may write

$$
\begin{aligned}
& \left|\left(\Phi_{1} y_{1}\right)(t)-\left(\Phi_{1} y_{2}\right)(t)\right| \\
& =\mid \Psi\left(t, y_{1}(t), \lambda_{2}^{*} \int_{1}^{e} y_{1}(\varpi) \mathrm{d} \varpi,{ }^{\mathcal{H}} \mathcal{I}_{1^{+}}^{\mu^{*}} y_{1}(t)\right) \\
& \quad-\Psi\left(t, y_{2}(t), \lambda_{2}^{*} \int_{1}^{e} y_{2}(\varpi) \mathrm{d} \varpi,{ }^{\mathcal{H}} \mathcal{I}_{1^{+}}^{\mu^{*}} y_{2}(t)\right) \mid \\
& \leq \varrho(t)\left[1+\left|\lambda_{2}^{*}(e-1)\right|+\frac{1}{\Gamma\left(\mu^{*}+1\right)}\right] \sup _{t \in[1, e]}\left|y_{1}(t)-y_{2}(t)\right|
\end{aligned}
$$

for any $t \in[1, e]$. Hence, we get

$$
\left\|\Phi_{1} y_{1}-\Phi_{1} y_{2}\right\| \mathcal{X} \leq \varrho^{*}\left[1+\left|\lambda_{2}^{*}(e-1)\right|+\frac{1}{\Gamma\left(\mu^{*}+1\right)}\right]\left\|y_{1}-y_{2}\right\|_{\mathcal{X}}
$$


showing that $\Phi_{1}$ is Lipschitz with constant $\varrho^{*}\left[1+\left|\lambda_{2}^{*}(e-1)\right|+\frac{1}{\Gamma\left(\mu^{*}+1\right)}\right]>0$ for each $y_{1}, y_{2} \in \mathcal{X}$. Similarly, by using hypothesis $(\mathcal{H P} 2)$, one can realize that $\Phi_{1}$ is also Lipschitz on $\mathcal{X}$. The proof is straightforward as above. Indeed, we have

$$
\begin{aligned}
&\left|\left(\Phi_{2} y_{1}\right)(t)-\left(\Phi_{2} y_{2}\right)(t)\right| \\
&=\mid \Lambda\left(t, y_{1}(t), \lambda_{1}^{*} \int_{1}^{e} y_{1}(\varpi) \mathrm{d} \varpi,{ }^{\mathcal{H}} \mathcal{I}_{1^{+}}^{\gamma^{*}} y_{1}(t)\right) \\
& \quad-\Lambda\left(t, y_{2}(t), \lambda_{1}^{*} \int_{1}^{e} y_{2}(\varpi) \mathrm{d} \varpi,{ }^{\mathcal{H}} \mathcal{I}_{1^{+}}^{\gamma^{*}} y_{2}(t)\right) \mid \\
& \leq \sigma(t)\left[1+\left|\lambda_{1}^{*}(e-1)\right|+\frac{1}{\Gamma\left(\gamma^{*}+1\right)}\right] \sup _{t \in[1, e]}\left|y_{1}(t)-y_{2}(t)\right|
\end{aligned}
$$

for any $t \in[1, e]$. Thus, we get

$$
\left\|\Phi_{2} y_{1}-\Phi_{2} y_{2}\right\| \mathcal{X} \leq \sigma^{*}\left[1+\left|\lambda_{1}^{*}(e-1)\right|+\frac{1}{\Gamma\left(\gamma^{*}+1\right)}\right]\left\|y_{1}-y_{2}\right\| \mathcal{X}
$$

demonstrating that $\Phi_{2}$ is Lipschitz with constant $\sigma^{*}\left[1+\left|\lambda_{1}^{*}(e-1)\right|+\frac{1}{\Gamma\left(\gamma^{*}+1\right)}\right]>0$ for each $y_{1}, y_{2} \in \mathcal{X}$. Therefore, the first condition of Theorem 5 is fulfilled for two operators $\Phi_{1}$ and $\Phi_{2}$. In the sequel, we establish the complete continuity of the operator $\Phi_{3}$ on the given closed ball $\overline{\mathcal{V}_{\rho}(0)}$. We have to check that $\Phi_{3}$ is continuous on $\overline{\mathcal{V}_{\rho}(0)}$. Thus, consider a convergent sequence $\left\{y_{n}\right\}$ in $\overline{\mathcal{V}_{\rho}(0)}$ so that $y_{n} \rightarrow y$, where $y \in \overline{\mathcal{V}_{\rho}(0)}$ is an arbitrary element. By assumption, we know that $\hat{\Upsilon}$ is continuous on $[1, e] \times \mathcal{X}$, so $\lim _{n \rightarrow \infty} \hat{\Upsilon}\left(t, y_{n}\right)=\hat{\Upsilon}(t, y)$. By Lebesgue's dominated convergence theorem, we obtain

$$
\begin{aligned}
\lim _{n \rightarrow \infty}\left(\Phi_{3} y_{n}\right)(t)= & \frac{1}{\Gamma\left(\kappa^{*}\right)} \int_{1}^{t}\left(\ln \frac{t}{\varpi}\right)^{\kappa^{*}-1} \lim _{n \rightarrow \infty} \hat{\Upsilon}\left(\varpi, y_{n}(\varpi)\right) \frac{\mathrm{d} \varpi}{\varpi} \\
& +\frac{\tilde{a_{2}}\left(1+\tilde{a_{1}} \ln (t)\right)}{\mathcal{Q}^{*} \Gamma\left(\kappa^{*}\right)} \int_{1}^{e}\left(\ln \frac{e}{\varpi}\right)^{\kappa^{*}-1} \lim _{n \rightarrow \infty} \hat{\Upsilon}\left(\varpi, y_{n}(\varpi)\right) \frac{\mathrm{d} \varpi}{\varpi} \\
& -\frac{\left(1+\tilde{a_{1}} \ln (t)\right)}{\mathcal{Q}^{*} \Gamma\left(\kappa^{*}+\theta^{*}-1\right)} \int_{1}^{e}\left(\ln \frac{e}{\varpi}\right)^{\kappa^{*}+\theta^{*}-2} \lim _{n \rightarrow \infty} \hat{\Upsilon}\left(\varpi, y_{n}(\varpi)\right) \frac{\mathrm{d} \varpi}{\varpi} \\
= & \frac{1}{\Gamma\left(\kappa^{*}\right)} \int_{1}^{t}\left(\ln \frac{t}{\varpi}\right)^{\kappa^{*}-1} \hat{\Upsilon}(\varpi, y(\varpi)) \frac{\mathrm{d} \varpi}{\varpi} \\
& +\frac{\tilde{a_{2}}\left(1+\tilde{a_{1}} \ln (t)\right)}{\mathcal{Q}^{*} \Gamma\left(\kappa^{*}\right)} \int_{1}^{e}\left(\ln \frac{e}{\varpi}\right)^{\kappa^{*}-1} \hat{\Upsilon}(\varpi, y(\varpi)) \frac{\mathrm{d} \varpi}{\varpi} \\
& -\frac{\left(1+\tilde{a_{1}} \ln (t)\right)}{\mathcal{Q}^{*} \Gamma\left(\kappa^{*}+\theta^{*}-1\right)} \int_{1}^{e}\left(\ln \frac{e}{\varpi}\right)^{\kappa^{*}+\theta^{*}-2} \hat{\Upsilon}(\varpi, y(\varpi)) \frac{\mathrm{d} \varpi}{\varpi}=\left(\Phi_{3} y\right)(t)
\end{aligned}
$$

for any $t \in[1, e]$. Hence, we get $\Phi_{3} y_{n} \rightarrow \Phi_{3} y$ as $n \rightarrow \infty$, and this means that $\Phi_{3}$ is continuous on $\overline{\mathcal{V}_{\rho}(0)}$. The next goal is to check the uniform boundedness of $\Phi_{3}$ on the ball $\overline{\mathcal{V}_{\rho}(0)}$. Let us take $y \in \overline{\mathcal{V}_{\rho}(0)}$. Under hypothesis $(\mathcal{H P} 3)$, the following estimate is obtained:

$$
\begin{aligned}
\left|\left(\Phi_{3} y\right)(t)\right| \leq & \frac{1}{\Gamma\left(\kappa^{*}\right)} \int_{1}^{t}\left(\ln \frac{t}{\varpi}\right)^{\kappa^{*}-1}|\hat{\Upsilon}(\varpi, y(\varpi))| \frac{\mathrm{d} \varpi}{\varpi} \\
& +\left|\frac{\tilde{a_{2}}\left(1+\tilde{a_{1}}\right)}{\mathcal{Q}^{*} \Gamma\left(\kappa^{*}\right)}\right| \int_{1}^{e}\left(\ln \frac{e}{\varpi}\right)^{\kappa^{*}-1}|\hat{\Upsilon}(\varpi, y(\varpi))| \frac{\mathrm{d} \varpi}{\varpi}
\end{aligned}
$$




$$
\begin{aligned}
& +\left|\frac{\left(1+\tilde{a_{1}}\right)}{\mathcal{Q}^{*} \Gamma\left(\kappa^{*}+\theta^{*}-1\right)}\right| \int_{1}^{e}\left(\ln \frac{e}{\varpi}\right)^{\kappa^{*}+\theta^{*}-2}|\hat{\Upsilon}(\varpi, y(\varpi))| \frac{\mathrm{d} \varpi}{\varpi} \\
\leq & \frac{\sup _{t \in[1, e]} \psi(t) \times \xi\left(\|y\|_{\mathcal{X}}\right)}{\Gamma\left(\kappa^{*}+1\right)}+\left|\frac{\tilde{a_{2}}\left(1+\tilde{a_{1}}\right)}{\mathcal{Q}^{*} \Gamma\left(\kappa^{*}+1\right)}\right| \sup _{t \in[1, e]} \psi(t) \times \xi\left(\|y\|_{\mathcal{X}}\right) \\
& +\left|\frac{\left(1+\tilde{a_{1}}\right)}{\mathcal{Q}^{*} \Gamma\left(\kappa^{*}+\theta^{*}\right)}\right| \sup _{t \in[1, e]} \psi(t) \times \xi\left(\|y\|_{\mathcal{X}}\right)
\end{aligned}
$$

for each $t \in[1, e]$. It follows that $\left\|\left(\Phi_{3} y\right)(t)\right\|_{\mathcal{X}} \leq \psi^{*} \xi\left(\|y\|_{\mathcal{X}}\right)$. Hence $\Phi_{3}\left(\overline{\mathcal{V}_{\rho}(0)}\right)$ is a uniformly bounded subset of $\mathcal{X}$. To establish the complete continuity property of $\Phi_{3}$ in the last step, it is enough to verify the equicontinuity of $\Phi_{3}$. For this purpose, we take two arbitrary elements $t_{1}, t_{2} \in[1, e]$ so that $t_{1}<t_{2}$ and $y \in \overline{\mathcal{V}_{\rho}(0)}$. Then, under appropriate conditions, we can write

$$
\begin{aligned}
\left|\left(\Phi_{3} y\right)\left(t_{2}\right)-\left(\Phi_{3} y\right)\left(t_{1}\right)\right| & \leq \frac{1}{\Gamma\left(\kappa^{*}\right)} \int_{1}^{t_{1}}\left[\left(\ln \frac{t_{2}}{\varpi}\right)^{\kappa^{*}-1}-\left(\ln \frac{t_{1}}{\varpi}\right)^{\kappa^{*}-1}\right]|\hat{\Upsilon}(\varpi, y(\varpi))| \frac{\mathrm{d} \varpi}{\varpi} \\
& +\frac{1}{\Gamma\left(\kappa^{*}\right)} \int_{t_{1}}^{t_{2}}\left(\ln \frac{t_{2}}{\varpi}\right)^{\kappa^{*}-1}|\hat{\Upsilon}(\varpi, y(\varpi))| \frac{\mathrm{d} \varpi}{\varpi} \\
& +\frac{\left|\tilde{a_{2}} \tilde{a}_{1}\right|\left(\left|\ln \left(t_{2}\right)-\ln \left(t_{1}\right)\right|\right)}{\left|\mathcal{Q}^{*}\right| \Gamma\left(\kappa^{*}\right)} \int_{1}^{e}\left(\ln \frac{e}{\varpi}\right)^{\kappa^{*}-1}|\hat{\Upsilon}(\varpi, y(\varpi))| \frac{\mathrm{d} \varpi}{\varpi} \\
& +\frac{\left|\tilde{a_{1}}\right|\left(\left|\ln \left(t_{2}\right)-\ln \left(t_{1}\right)\right|\right)}{\left|\mathcal{Q}^{*}\right| \Gamma\left(\kappa^{*}+\theta^{*}-1\right)} \int_{1}^{e}\left(\ln \frac{e}{\varpi}\right)^{\kappa^{*}+\theta^{*}-2}|\hat{\Upsilon}(\varpi, y(\varpi))| \frac{\mathrm{d} \varpi}{\varpi} \\
\leq & \frac{\left(\left(\ln \left(t_{2}\right)\right)^{k^{*}}-\left(\ln \left(t_{1}\right)\right)^{k^{*}}\right)-\left(\ln \left(t_{2}\right)-\ln \left(t_{1}\right)\right)^{k^{*}}}{\Gamma\left(\kappa^{*}+1\right)} \sup _{t \in[1, e]} \psi(t) \times \xi(\|y\| \mathcal{X}) \\
& +\frac{\left(\ln \left(t_{2}\right)-\ln \left(t_{1}\right)\right)^{k^{*}}}{\Gamma\left(\kappa^{*}+1\right)} \sup _{t \in[1, e]} \psi(t) \times \xi(\|y\| \mathcal{X}) \\
& +\frac{\left|\tilde{a_{2}} \tilde{a}_{1}\right|\left(\left|\ln \left(t_{2}\right)-\ln \left(t_{1}\right)\right|\right)}{\left|\mathcal{Q}^{*}\right| \Gamma\left(\kappa^{*}\right)} \int_{1}^{e}\left(\ln \frac{e}{\varpi}\right)^{\kappa^{*}-1}|\hat{\Upsilon}(\varpi, y(\varpi))| \frac{\mathrm{d} \varpi}{\varpi} \\
& +\frac{\left|\tilde{a_{1}}\right|\left(\left|\ln \left(t_{2}\right)-\ln \left(t_{1}\right)\right|\right)}{\left|\mathcal{Q}^{*}\right| \Gamma\left(\kappa^{*}+\theta^{*}-1\right)} \int_{1}^{e}\left(\ln \frac{e}{\varpi}\right)^{\kappa^{*}+\theta^{*}-2}|\hat{\Upsilon}(\varpi, y(\varpi))| \frac{\mathrm{d} \varpi}{\varpi} .
\end{aligned}
$$

When we take the limit on both sides of the inequality as $t_{1} \rightarrow t_{2}$, then clearly the RHS of the inequality approaches 0 (regardless of $\left.y \in \overline{\mathcal{V}_{\rho}(0)}\right)$. Thus, $\left|\left(\Phi_{3} y\right)\left(t_{2}\right)-\left(\Phi_{3} y\right)\left(t_{1}\right)\right| \rightarrow 0$ as $t_{1} \rightarrow t_{2}$, confirming the equicontinuity of the operator $\Phi_{3}$. Here, by invoking the ArzelaAscoli theorem, it is deduced that $\Phi_{3}$ is completely continuous on $\overline{\mathcal{V}}_{\rho}(0)$. To fulfill the third condition of Theorem 5 , we utilize hypothesis $(\mathcal{H P} 3)$ and obtain

$$
\begin{aligned}
\hat{\Delta} & =\| \Phi_{3}\left(\overline{\left.\mathcal{V}_{\rho}(0)\right)} \|_{\mathcal{X}}\right. \\
& =\sup _{t \in[1, e]}\left\{\left|\left(\Phi_{3} y\right)(t)\right|: y \in \overline{\mathcal{V}_{\rho}(0)}\right\} \\
& \leq \psi^{*} \xi\left(\|y\|_{\mathcal{X}}\right)\left(\frac{1}{\Gamma\left(\kappa^{*}+1\right)}+\left|\frac{\tilde{a_{2}}\left(1+\tilde{a_{1}}\right)}{\mathcal{Q}^{*} \Gamma\left(\kappa^{*}+1\right)}\right|+\left|\frac{\left(1+\tilde{a_{1}}\right)}{\mathcal{Q}^{*} \Gamma\left(\kappa^{*}+\theta^{*}\right)}\right|\right) \\
& =\psi^{*} \xi\left(\|y\|_{\mathcal{X}}\right) \tilde{M} .
\end{aligned}
$$


Hence $\hat{\Delta} \leq \psi^{*} \xi\left(\|y\|_{\mathcal{X}}\right) \tilde{M}$. Therefore we have

$$
\begin{aligned}
\varrho^{*} & {\left[1+\left|\lambda_{2}^{*}(e-1)\right|+\frac{1}{\Gamma\left(\mu^{*}+1\right)}\right] \hat{\Delta}+\sigma^{*}\left[1+\left|\lambda_{1}^{*}(e-1)\right|+\frac{1}{\Gamma\left(\gamma^{*}+1\right)}\right] } \\
\leq & \varrho^{*}\left[1+\left|\lambda_{2}^{*}(e-1)\right|+\frac{1}{\Gamma\left(\mu^{*}+1\right)}\right] \psi^{*} \xi(\|y\| \mathcal{X}) \tilde{M} \\
& +\sigma^{*}\left[1+\left|\lambda_{1}^{*}(e-1)\right|+\frac{1}{\Gamma\left(\gamma^{*}+1\right)}\right]<1 .
\end{aligned}
$$

At this point, setting $\tilde{K}_{1}^{*}=\varrho^{*}\left[1+\left|\lambda_{2}^{*}(e-1)\right|+\frac{1}{\Gamma\left(\mu^{*}+1\right)}\right]$ and $\tilde{K}_{2}^{*}=\sigma^{*}\left[1+\left|\lambda_{1}^{*}(e-1)\right|+\frac{1}{\Gamma\left(\gamma^{*}+1\right)}\right]$, we reach $\tilde{K}_{1}^{*} \hat{\Delta}+\tilde{K}_{2}^{*}<1$. So far, all three hypotheses of Theorem 5 are fulfilled. Thus in the following, we claim that one of the conditions (a) or (b) in Theorem 5 is possible. To begin, we check condition (b). Let $\alpha_{0} \in(0,1)$ and suppose that there exists $y \in \mathcal{X}$ with $\|y\|_{\mathcal{X}}=\rho$ so that the equation $y=\alpha_{0}\left(\Phi_{1} y\right)\left(\Phi_{3} y\right)+\alpha_{0}\left(\Phi_{2} y\right)$ holds. Then, we have

$$
\begin{aligned}
& |y(t)| \leq \alpha_{0}\left|\left(\Phi_{1} y\right)(t)\right|\left|\left(\Phi_{3} y\right)(t)\right|+\alpha_{0}\left|\left(\Phi_{2} y\right)(t)\right| \\
& \leq \alpha_{0}\left|\Psi\left(t, y(t), \lambda_{2}^{*} \int_{1}^{e} y(\varpi) \mathrm{d} \varpi,{ }^{\mathcal{H}} \mathcal{I}_{1^{+}}^{\mu^{*}} y(t)\right)\right| \\
& \times\left(\mid \frac{1}{\Gamma\left(\kappa^{*}\right)} \int_{1}^{t}\left(\ln \frac{t}{\varpi}\right)^{\kappa^{*}-1} \hat{\Upsilon}(\varpi, y(\varpi)) \frac{\mathrm{d} \varpi}{\varpi}\right. \\
& +\frac{\tilde{a_{2}}\left(1+\tilde{a_{1}} \ln (t)\right)}{\mathcal{Q}^{*} \Gamma\left(\kappa^{*}\right)} \int_{1}^{e}\left(\ln \frac{e}{\varpi}\right)^{\kappa^{*}-1} \hat{\Upsilon}(\varpi, y(\varpi)) \frac{\mathrm{d} \varpi}{\varpi} \\
& -\frac{\left(1+\tilde{a_{1}} \ln (t)\right)}{\mathcal{Q}^{*} \Gamma\left(\kappa^{*}+\theta^{*}-1\right)} \\
& \left.\times \int_{1}^{e}\left(\ln \frac{e}{\varpi}\right)^{\kappa^{*}+\theta^{*}-2} \hat{\Upsilon}(\varpi, y(\varpi)) \frac{\mathrm{d} \varpi}{\varpi} \mid\right) \\
& +\alpha_{0}\left|\Lambda\left(t, y(t), \lambda_{1}^{*} \int_{1}^{e} y(\varpi) \mathrm{d} \varpi,{ }^{\mathcal{H}} \mathcal{I}_{1^{+}}^{\gamma^{*}} y(t)\right)\right| \\
& \leq\left|\Psi\left(t, y(t), \lambda_{2}^{*} \int_{1}^{e} y(\varpi) \mathrm{d} \varpi,{ }^{\mathcal{H}} \mathcal{I}_{1^{+}}^{\mu^{*}} y(t)\right)-\Psi(t, 0,0,0)+\Psi(t, 0,0,0)\right| \\
& \times\left(\frac{\sup _{t \in[1, e]} \psi(t) \times \xi\left(\|y\|_{\mathcal{X}}\right)}{\Gamma\left(\kappa^{*}+1\right)}+\sup _{t \in[1, e]} \psi(t) \times \xi\left(\|y\|_{\mathcal{X}}\right)\left|\frac{\tilde{a_{2}}\left(1+\tilde{a_{1}}\right)}{\mathcal{Q}^{*} \Gamma\left(\kappa^{*}+1\right)}\right|\right. \\
& \left.+\sup _{t \in[1, e]} \psi(t) \times \xi(\|y\| \mathcal{X})\left|\frac{\left(1+\tilde{a_{1}}\right)}{\mathcal{Q}^{*} \Gamma\left(\kappa^{*}+\theta^{*}\right)}\right|\right) \\
& +\left|\Lambda\left(t, y(t), \lambda_{1}^{*} \int_{1}^{e} y(\varpi) \mathrm{d} \varpi,{ }^{\mathcal{H}} \mathcal{I}_{1^{+}}^{\gamma^{*}} y(t)-\Lambda(t, 0,0,0)|+| \Lambda(t, 0,0,0)\right)\right| \\
& \leq\left[\varrho^{*}\left(1+\left|\lambda_{2}^{*}(e-1)\right|+\frac{1}{\Gamma\left(\mu^{*}+1\right)}\right)\|y\|_{\mathcal{X}}+\Psi^{*}\right] \tilde{M} \psi^{*} \xi\left(\|y\|_{\mathcal{X}}\right) \\
& +\sigma^{*}\left(1+\left|\lambda_{1}^{*}(e-1)\right|+\frac{1}{\Gamma\left(\gamma^{*}+1\right)}\right)\|y\|_{\mathcal{X}}+\Lambda^{*} .
\end{aligned}
$$


So, we arrive at the following inequality:

$$
\begin{aligned}
\rho= & \|y(t)\|_{\mathcal{X}} \\
\leq & {\left[\varrho^{*}\left(1+\left|\lambda_{2}^{*}(e-1)\right|+\frac{1}{\Gamma\left(\mu^{*}+1\right)}\right) \rho+\tilde{M} \Psi^{*}\right] \psi^{*} \xi(\rho) } \\
& +\sigma^{*}\left(1+\left|\lambda_{1}^{*}(e-1)\right|+\frac{1}{\Gamma\left(\gamma^{*}+1\right)}\right) \rho+\Lambda^{*} .
\end{aligned}
$$

This implies that

$$
\rho \leq \frac{\tilde{M} \psi^{*} \xi(\rho) \Psi^{*}+\Lambda^{*}}{1-\varrho^{*}\left(1+\left|\lambda_{2}^{*}(e-1)\right|+\frac{1}{\Gamma\left(\mu^{*}+1\right)}\right) \tilde{M} \psi^{*} \xi(\rho)-\sigma^{*}\left(1+\left|\lambda_{1}^{*}(e-1)\right|+\frac{1}{\Gamma\left(\gamma^{*}+1\right)}\right)}
$$

which is impossible due to (9). Therefore, condition (b) stated in Theorem 5 is not fulfilled and so condition (a) in Theorem 5 holds. Consequently, the operator equation $\left(\Phi_{1} y\right)\left(\Phi_{3} y\right)+\left(\Phi_{2} y\right)=y$ has a solution. This means that the mixed Caputo-Hadamard hybrid BVP (1)-(2) has at least one solution.

\subsection{Special cases}

This subsection is devoted to deriving some analytical existence criteria for a special case formulated by mixed Caputo-Hadamard nonhybrid BVP (3). We state some hypotheses as follows:

$(\mathcal{H P} 5)$ (Lipschitz property) There exists a constant $L_{\hat{\Upsilon}}>0$ such that for each $y, y^{\prime} \in \mathcal{X}$, we have $\left|\hat{\Upsilon}(t, y)-\hat{\Upsilon}\left(t, y^{\prime}\right)\right| \leq L_{\hat{\Upsilon}}\left|y-y^{\prime}\right|$.

(HP 6) (Boundedness property) There are constants $C_{\hat{\gamma}}$ and $M_{\hat{\gamma}}$ such that for each $y \in \mathbb{R}$ we have $|\hat{\Upsilon}(t, y)| \leq C_{\hat{\gamma}}|y|+M_{\hat{\Upsilon}}$.

$(\mathcal{H P} 7)$ One has $\frac{L_{\hat{\gamma}}}{\Gamma\left(\kappa^{*}+1\right)}<1$.

In the following lemma, an integral structure of the solution for the mixed CaputoHadamard BVP (3) is demonstrated.

Lemma 9 Let $g \in \mathcal{X}$. Then a function $\tilde{y}_{0}$ is a solution for two-point Caputo-Hadamard fractional differential equation with mixed Hadamard integral boundary conditions

$$
\left\{\begin{array}{l}
\mathcal{C H} \mathcal{D}_{1^{+}}^{\kappa^{*}} y(t)=g(t), \\
\mathcal{C H}^{\mathcal{D}_{1^{+}}^{\kappa^{*}} y(1)}=\tilde{a_{1}} y(1), \quad \frac{1}{\Gamma\left(\theta^{*}\right)} \int_{1}^{e}\left(\ln \frac{e}{\varpi} \theta^{\theta^{*}-1}\left[{ }^{\mathcal{H}} \mathcal{D}_{1^{+}}^{\kappa^{*}} y(\varpi)\right] \frac{\mathrm{d} \omega}{\varpi}=\tilde{a_{2}} y(e)\right.
\end{array}\right.
$$

if and only if $\tilde{y}_{0}$ is a solution of the Hadamard fractional integral equation

$$
\begin{aligned}
y(t)= & \frac{1}{\Gamma\left(\kappa^{*}\right)} \int_{1}^{t}\left(\ln \frac{t}{\varpi}\right)^{\kappa^{*}-1} g(\varpi) \frac{\mathrm{d} \varpi}{\varpi}+\frac{\tilde{a_{2}}\left(1+\tilde{a_{1}} \ln (t)\right)}{\mathcal{Q}^{*} \Gamma\left(\kappa^{*}\right)} \int_{1}^{e}\left(\ln \frac{e}{\varpi}\right)^{\kappa^{*}-1} g(\varpi) \frac{\mathrm{d} \varpi}{\varpi} \\
& -\frac{\left(1+\tilde{a_{1}} \ln (t)\right)}{\mathcal{Q}^{*} \Gamma\left(\kappa^{*}+\theta^{*}-1\right)} \int_{1}^{e}\left(\ln \frac{e}{\varpi}\right)^{\kappa^{*}+\theta^{*}-2} g(\varpi) \frac{\mathrm{d} \varpi}{\varpi},
\end{aligned}
$$

where $\mathcal{Q}^{*}$ is given by (7).

Proof The proof is similar to that of Lemma 7 and so is omitted. 
We define an operator $\Phi: \mathcal{X} \rightarrow \mathcal{X}$ by $\Phi y(t)=\Phi_{1} y(t)+\Phi_{2} y(t)$ which splits into two operators $\Phi_{1}: \mathcal{X} \rightarrow \mathcal{X}$ and $\Phi_{2}: \mathcal{X} \rightarrow \mathcal{X}$ as follows:

$$
\begin{aligned}
\Phi_{1} y(t)= & \frac{1}{\Gamma\left(\kappa^{*}\right)} \int_{1}^{t}\left(\ln \frac{t}{\varpi}\right)^{\kappa^{*}-1} \hat{\Upsilon}(\varpi, y(\varpi)) \frac{\mathrm{d} \varpi}{\varpi}, \\
\Phi_{2} y(t)= & \frac{\tilde{a}_{2}\left(1+\tilde{a_{1}} \ln (t)\right)}{\mathcal{Q}^{*} \Gamma\left(\kappa^{*}\right)} \int_{1}^{e}\left(\ln \frac{e}{\varpi}\right)^{\kappa^{*}-1} \hat{\Upsilon}(\varpi, y(\varpi)) \frac{\mathrm{d} \varpi}{\varpi} \\
& -\frac{\left(1+\tilde{a_{1}} \ln (t)\right)}{\mathcal{Q}^{*} \Gamma\left(\kappa^{*}+\theta^{*}-1\right)} \int_{1}^{e}\left(\ln \frac{e}{\varpi}\right)^{\kappa^{*}+\theta^{*}-2} \hat{\Upsilon}(\varpi, y(\varpi)) \frac{\mathrm{d} \varpi}{\varpi},
\end{aligned}
$$

for each $y \in \mathcal{X}$ and $t \in[1, e]$. In this case, the equivalence of the existence of a solution for Caputo-Hadamard BVP (3) and the existence of a fixed point for operator $\Phi$ is obvious. Note that in all the following lemmas, we assume that $\mathcal{X}$ is a Banach space with sup-norm $\|\cdot\|_{\mathcal{X}}$ and two operators $\Phi_{1}$ and $\Phi_{2}$ are defined as in (10) and (11).

Lemma 10 Under hypothesis $(\mathcal{H P} 5)$, the operator $\Phi_{1}$ is Lipschitz with constant $\tilde{K}_{1}^{*}=$ $\frac{L_{\hat{\gamma}}}{\Gamma\left(\kappa^{*}+1\right)}$ and the following growth condition holds:

$$
\left\|\Phi_{1}(y)\right\|_{\mathcal{X}} \leq \frac{C_{\hat{\Upsilon}}}{\Gamma\left(\kappa^{*}+1\right)}\|y\|_{\mathcal{X}}+\frac{M_{\hat{\gamma}}}{\Gamma\left(\kappa^{*}+1\right)}
$$

for all $y \in \mathcal{X}$.

Proof By utilizing assumption $(\mathcal{H} \mathcal{P} 5)$, we obtain

$$
\begin{aligned}
& \left|\Phi_{1} y(t)-\Phi_{1} y^{\prime}(t)\right| \\
& \quad=\frac{1}{\Gamma\left(\kappa^{*}\right)}\left|\int_{1}^{t}\left(\ln \frac{t}{\varpi}\right)^{\kappa^{*}-1} \hat{\Upsilon}(\varpi, y(\varpi)) \frac{\mathrm{d} \varpi}{\varpi}-\int_{1}^{t}\left(\ln \frac{t}{\varpi}\right)^{\kappa^{*}-1} \hat{\Upsilon}\left(\varpi, y^{\prime}(\varpi)\right) \frac{\mathrm{d} \varpi}{\varpi}\right| \\
& \quad \leq \frac{L_{\hat{\Upsilon}}}{\Gamma\left(\kappa^{*}+1\right)}\left\|y-y^{\prime}\right\| .
\end{aligned}
$$

This implies that $\Phi_{1}$ is Lipschitz with constant $\tilde{K}_{1}^{*}=\frac{L_{\hat{\gamma}}}{\Gamma\left(\kappa^{*}+1\right)}$. Hence by Proposition 2, it is deduced that $\Phi_{1}$ is also $\mu$-Lipschitz with the same constant $\tilde{K}_{1}^{*}=\frac{L_{\hat{\gamma}}}{\Gamma\left(\kappa^{*}+1\right)}$, where $\mu$ is the Kuratowski's measure of noncompactness. Again, by considering $(\mathcal{H} \mathcal{P} 5)$ for the growth condition, we get

$$
\left\|\Phi_{1}(y)\right\|_{\mathcal{X}} \leq \frac{C_{\hat{\Upsilon}}}{\Gamma\left(\kappa^{*}+1\right)}\|y\|_{\mathcal{X}}+\frac{M_{\hat{\Upsilon}}}{\Gamma\left(\kappa^{*}+1\right)}
$$

and the proof is concluded.

Lemma 11 Operator $\Phi_{2}$ is continuous and also, in view of hypothesis $(\mathcal{H P} 6)$, we have the growth condition $\left\|\Phi_{2}(y)\right\|_{\mathcal{X}} \leq \Delta_{1}\|y\|_{\mathcal{X}}+\Delta_{2}$ for every $y \in \mathcal{X}$, where $\Delta_{1}=C_{\hat{\Upsilon}}\left(\left|\frac{\tilde{a_{2}}\left(1+\tilde{a}_{1}\right)}{\mathcal{Q}^{*} \Gamma\left(\kappa^{*}+1\right)}\right|+\right.$ $\left.\left|\frac{\left(1+\tilde{a_{1}}\right)}{\mathcal{Q}^{*} \Gamma\left(\kappa^{*}+\theta^{*}\right)}\right|\right)$ and

$$
\Delta_{2}=M_{\hat{\Upsilon}}\left(\left|\frac{\tilde{a_{2}}\left(1+\tilde{a_{1}}\right)}{\mathcal{Q}^{*} \Gamma\left(\kappa^{*}+1\right)}\right|+\left|\frac{\left(1+\tilde{a_{1}}\right)}{\mathcal{Q}^{*} \Gamma\left(\kappa^{*}+\theta^{*}\right)}\right|\right) .
$$


Proof By assumption, we know that $\hat{\Upsilon}$ is continuous on $[1, e] \times \mathcal{X}$, and so we conclude that $\lim _{n \rightarrow \infty} \hat{\Upsilon}\left(t, y_{n}\right)=\hat{\Upsilon}(t, y)$. By invoking the Lebesgue's dominated convergence theorem, we obtain

$$
\begin{aligned}
\lim _{n \rightarrow \infty}\left(\Phi_{2} y_{n}\right)(t)= & \frac{\tilde{a}_{2}\left(1+\tilde{a_{1}} \ln (t)\right)}{\mathcal{Q}^{*} \Gamma\left(\kappa^{*}\right)} \int_{1}^{e}\left(\ln \frac{e}{\varpi}\right)^{\kappa^{*}-1} \lim _{n \rightarrow \infty} \hat{\Upsilon}\left(\varpi, y_{n}(\varpi)\right) \frac{\mathrm{d} \varpi}{\varpi} \\
& -\frac{\left(1+\tilde{a_{1}} \ln (t)\right)}{\mathcal{Q}^{*} \Gamma\left(\kappa^{*}+\theta^{*}-1\right)} \int_{1}^{e}\left(\ln \frac{e}{\varpi}\right)^{\kappa^{*}+\theta^{*}-2} \lim _{n \rightarrow \infty} \hat{\Upsilon}\left(\varpi, y_{n}(\varpi)\right) \frac{\mathrm{d} \varpi}{\varpi} \\
= & \frac{\tilde{a}_{2}\left(1+\tilde{a_{1}} \ln (t)\right)}{\mathcal{Q}^{*} \Gamma\left(\kappa^{*}\right)} \int_{1}^{e}\left(\ln \frac{e}{\varpi}\right)^{\kappa^{*}-1} \hat{\Upsilon}(\varpi, y(\varpi)) \frac{\mathrm{d} \varpi}{\varpi} \\
& -\frac{\left(1+\tilde{a_{1}} \ln (t)\right)}{\mathcal{Q}^{*} \Gamma\left(\kappa^{*}+\theta^{*}-1\right)} \int_{1}^{e}\left(\ln \frac{e}{\varpi}\right)^{\kappa^{*}+\theta^{*}-2} \hat{\Upsilon}(\varpi, y(\varpi)) \frac{\mathrm{d} \varpi}{\varpi}=\left(\Phi_{2} y\right)(t)
\end{aligned}
$$

for any $t \in[1, e]$. Hence, we see that $\Phi_{2} y_{n} \rightarrow \Phi_{2} y$ as $n \rightarrow \infty$, and so $\Phi_{2}$ is continuous on $\overline{\mathcal{V}_{\rho}(0)}$. Now, for the sake of the investigation of the growth condition on $\Phi_{2}$, we utilize hypothesis $(\mathcal{H P} 6)$ and obtain

$$
\begin{aligned}
\left|\Phi_{2} y(t)\right|= & \mid \frac{\tilde{a_{2}}\left(1+\tilde{a_{1}} \ln (t)\right)}{\mathcal{Q}^{*} \Gamma\left(\kappa^{*}\right)} \int_{1}^{e}\left(\ln \frac{e}{\varpi}\right)^{\kappa^{*}-1} \hat{\Upsilon}(\varpi, y(\varpi)) \frac{\mathrm{d} \varpi}{\varpi} \\
& -\frac{\left(1+\tilde{a_{1}} \ln (t)\right)}{\mathcal{Q}^{*} \Gamma\left(\kappa^{*}+\theta^{*}-1\right)} \int_{1}^{e}\left(\ln \frac{e}{\varpi}\right)^{\kappa^{*}+\theta^{*}-2} \hat{\Upsilon}(\varpi, y(\varpi)) \frac{\mathrm{d} \varpi}{\varpi} \mid \\
\leq & \left|\frac{\tilde{a_{2}}\left(1+\tilde{a_{1}}\right)}{\mathcal{Q}^{*} \Gamma\left(\kappa^{*}\right)} \int_{1}^{e}\left(\ln \frac{e}{\varpi}\right)^{\kappa^{*}-1}\left(C_{\hat{\Upsilon}}|y(s)|+M_{\hat{\Upsilon}}\right) \frac{\mathrm{d} \varpi}{\varpi}\right| \\
& +\left|\frac{\left(1+\tilde{a_{1}}\right)}{\mathcal{Q}^{*} \Gamma\left(\kappa^{*}+\theta^{*}-1\right)} \int_{1}^{e}\left(\ln \frac{e}{\varpi}\right)^{\kappa^{*}+\theta^{*}-2}\left(C_{\hat{\Upsilon}}|y(s)|+M_{\hat{\Upsilon}}\right) \frac{\mathrm{d} \varpi}{\varpi}\right| \\
\leq & C_{\hat{\Upsilon}}\left(\left|\frac{\tilde{a_{2}}\left(1+\tilde{a_{1}}\right)}{\mathcal{Q}^{*} \Gamma\left(\kappa^{*}+1\right)}\right|+\left|\frac{\left(1+\tilde{a_{1}}\right)}{\mathcal{Q}^{*} \Gamma\left(\kappa^{*}+\theta^{*}\right)}\right|\right)\|y\| \mathcal{X} \\
& +M_{\hat{\Upsilon}}\left(\left|\frac{\tilde{a_{2}}\left(1+\tilde{a_{1}}\right)}{\mathcal{Q}^{*} \Gamma\left(\kappa^{*}+1\right)}\right|+\left|\frac{\left(1+\tilde{a_{1}}\right)}{\mathcal{Q}^{*} \Gamma\left(\kappa^{*}+\theta^{*}\right)}\right|\right)
\end{aligned}
$$

which is the desired conclusion.

Lemma 12 Operator $\Phi_{2}: \mathcal{X} \rightarrow \mathcal{X}$ is compact. Moreover, $\Phi_{2}$ is $\mu$-Lipschitz with constant $\tilde{K}_{2}^{*}=0$ where $\mu$ is the Kuratowski's measure of noncompactness.

Proof Consider a bounded subset $\mathcal{B} \subset \overline{\mathcal{V}_{\rho}(0)}$ in $\mathcal{X}$ and take a sequence $\left\{y_{n}\right\}$ belonging to $\mathcal{B}$. Then, by Lemma 11, we have

$$
\left\|\Phi_{2}\left(y_{n}\right)\right\|_{\mathcal{X}} \leq \Delta_{1}\left\|y_{n}\right\|_{\mathcal{X}}+\Delta_{2}<\infty
$$

for each $y_{n} \in \mathcal{B}$ which yields that $\Phi_{2}(\mathcal{B})$ is a bounded set. Besides, we verify that $\left\{\Phi_{2}\left(y_{n}\right)\right\}$ is equicontinuous for each $y_{n} \in \mathcal{B}$. Take $t_{1}, t_{2} \in[1, e]$ so that $t_{1}<t_{2}$. Then, we obtain

$$
\begin{aligned}
& \left|\Phi_{2}\left(y_{n}\right)\left(t_{2}\right)-\Phi_{2}\left(y_{n}\right)\left(t_{1}\right)\right| \\
& \quad \leq \frac{\left|\tilde{a_{2}}{\tilde{a_{1}}}_{1}\right|\left(\left|\ln \left(t_{2}\right)-\ln \left(t_{1}\right)\right|\right)}{\left|\mathcal{Q}^{*}\right| \Gamma\left(\kappa^{*}\right)} \int_{1}^{e}\left(\ln \frac{e}{\varpi}\right)^{\kappa^{*}-1}|\hat{\Upsilon}(\varpi, y(\varpi))| \frac{\mathrm{d} \varpi}{\varpi}
\end{aligned}
$$




$$
\begin{aligned}
& +\frac{\left|\tilde{a}_{1}\right|\left(\left|\ln \left(t_{2}\right)-\ln \left(t_{1}\right)\right|\right)}{\left|\mathcal{Q}^{*}\right| \Gamma\left(\kappa^{*}+\theta^{*}-1\right)} \int_{1}^{e}\left(\ln \frac{e}{\varpi}\right)^{\kappa^{*}+\theta^{*}-2}|\hat{\Upsilon}(\varpi, y(\varpi))| \frac{\mathrm{d} \varpi}{\varpi} \\
\leq & \frac{\left|\tilde{a_{2}} \tilde{a}_{1}\right|\left(\left|\ln \left(t_{2}\right)-\ln \left(t_{1}\right)\right|\right)}{\left|\mathcal{Q}^{*}\right| \Gamma\left(\kappa^{*}+1\right)}\left(C_{\hat{\Upsilon}}\|y\|_{\mathcal{X}}+M_{\hat{\Upsilon}}\right) \\
& +\frac{\left|\tilde{a}_{1}\right|\left(\left|\ln \left(t_{2}\right)-\ln \left(t_{1}\right)\right|\right)}{\left|\mathcal{Q}^{*}\right| \Gamma\left(\kappa^{*}+\theta^{*}\right)}\left(C_{\hat{\Upsilon}}\|y\|_{\mathcal{X}}+M_{\hat{\Upsilon}}\right) .
\end{aligned}
$$

Evidently, it is seen that the RHS of the inequality approaches 0 (regardless of the choice of $\left.y_{n} \in \mathcal{B}\right)$ whenever $t_{1} \rightarrow t_{2}$. Thus, letting $t_{1} \rightarrow t_{2}$, we get $\left|\Phi_{2}\left(y_{n}\right)\left(t_{2}\right)-\Phi_{2}\left(y_{n}\right)\left(t_{1}\right)\right| \rightarrow 0$ and so $\left\{\Phi_{2}\left(y_{n}\right)\right\}$ is equicontinuous. Taking into account the Arzela-Ascoli theorem, we obtain that $\Phi_{2}(\mathcal{B})$ is compact. In addition, in view of Proposition 3, $\Phi_{2}$ is $\mu$-Lipschitz with constant zero.

In this position, we establish the main results for the mixed Caputo-Hadamard nonhybrid BVP (3) based on the above lemmas.

Theorem 13 Under assumptions $(\mathcal{H P} 5)$ and $(\mathcal{H P} 6)$, the mixed Caputo-Hadamard nonhybrid BVP (3) has at least one solution $y \in \mathcal{X}$ provided $\frac{C_{\hat{r}}}{\Gamma\left(\kappa^{*}+1\right)}+\Delta_{1}<1$. Further, the family of solutions of (3) is bounded in the space $\mathcal{X}$.

Proof In view of the hypothesis $(\mathcal{H P} 7)$ and Lemma 10, we deduce that $\Phi_{1}: \mathcal{X} \rightarrow \mathcal{X}$ defined in (10) is $\mu$-Lipschitz with constant $\tilde{K}_{1}^{*}=\frac{L_{\hat{\gamma}}}{\Gamma\left(\kappa^{*}+1\right)} \in(0,1)$. Furthermore, we find that operator $\Phi_{2}: \mathcal{X} \rightarrow \mathcal{X}$ defined in (11) is $\mu$-Lipschitz with $\tilde{K}_{2}^{*}=0$ according to Lemma 12. Here, Proposition 4 implies that the operator $\Phi: \mathcal{X} \rightarrow \mathcal{X}$ defined by $\Phi=\Phi_{1}+\Phi_{2}$ is a strict $\mu$-contraction with constant $\tilde{K}^{*}=\tilde{K}_{1}^{*}+\tilde{K}_{2}^{*}=\tilde{K}_{1}^{*}$ and, since $\tilde{K}^{*}<1, \Phi$ is $\mu$-condensing. Now, take

$$
\mathcal{B}:=\{y \in \mathcal{X}: \text { there is } \lambda \in[0,1] \text { so that } y=\lambda \Phi(y)\} .
$$

In this step, it is enough to show that $\mathcal{B}$ is a bounded subset of $\mathcal{X}$. For this, select $y \in \mathcal{B}$. Then in the light of the growth conditions obtained in Lemmas 10 and 9 , we may write

$$
\begin{aligned}
\|y\|_{\mathcal{X}} & =\|\lambda \Phi(y)\|_{\mathcal{X}}=\lambda\|\Phi(y)\|_{\mathcal{X}} \leq \lambda\left(\left\|\Phi_{1}(y)\right\|_{\mathcal{X}}+\left\|\Phi_{2}(y)\right\|_{\mathcal{X}}\right) \\
& \leq \lambda\left(\frac{C_{\hat{\Upsilon}}}{\Gamma\left(\kappa^{*}+1\right)}\|y\|_{\mathcal{X}}+\frac{M_{\hat{\Upsilon}}}{\Gamma\left(\kappa^{*}+1\right)}+\Delta_{1}\|y\|_{\mathcal{X}}+\Delta_{2}\right) \\
& \leq \lambda\left(\frac{C_{\hat{\Upsilon}}}{\Gamma\left(\kappa^{*}+1\right)}+\Delta_{1}\right)\|y\|_{\mathcal{X}}+\lambda\left(\frac{M_{\hat{\gamma}}}{\Gamma\left(\kappa^{*}+1\right)}+\Delta_{2}\right),
\end{aligned}
$$

implying that the set $\mathcal{B}$ is bounded in $\mathcal{X}$. Thus there is a number $\rho>0$ such that $\mathcal{B} \subset$ $\overline{\mathcal{V}_{\rho}(0)}$, and so we have $\operatorname{deg}\left(I-\lambda \Phi, \overline{\mathcal{V}_{\rho}(0)}, 0\right)=1$, by applying Theorem 6 . Finally, under the hypotheses of Theorem 6 due to Isaia, the operator $\Phi=\Phi_{1}+\Phi_{2}$ has at least one fixed point and the family of fixed points of $\Phi$ is bounded in $\mathcal{X}$. This means that the mixed Caputo-Hadamard nonhybrid BVP (3) has at least one solution on $[1, e]$ and the family of solutions is bounded. The proof is finished.

Eventually, we derive a uniqueness criterion for the mixed Caputo-Hadamard nonhybrid BVP (3) in the following theorem. 


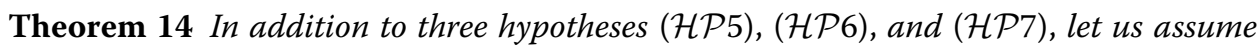
that

$$
L_{\hat{\Upsilon}}\left(\frac{1}{\Gamma\left(\kappa^{*}+1\right)}+\left|\frac{\tilde{a_{2}}\left(1+\tilde{a_{1}}\right)}{\mathcal{Q}^{*} \Gamma\left(\kappa^{*}+1\right)}\right|+\left|\frac{\left(1+\tilde{a_{1}}\right)}{\mathcal{Q}^{*} \Gamma\left(\kappa^{*}+\theta^{*}\right)}\right|\right)<1 .
$$

Then the mixed Caputo-Hadamard nonhybrid BVP (3) has a unique solution on $[1, e]$.

Proof Let $y \in \mathcal{X}$ be arbitrary. By Lemma 10 and assumption $(\mathcal{H P} 5)$, we obtain

$$
\left|\Phi_{1} y(t)-\Phi_{1} y^{\prime}(t)\right| \leq \frac{L_{\hat{\Upsilon}}}{\Gamma\left(\kappa^{*}+1\right)}\left\|y-y^{\prime}\right\|_{\mathcal{X}}
$$

where $\Phi_{1}: \mathcal{X} \rightarrow \mathcal{X}$ is defined in (10). Furthermore, we have the following estimate:

$$
\begin{aligned}
& \left|\Phi_{2} y(t)-\Phi_{2} y^{\prime}(t)\right| \\
& \leq\left|\frac{\tilde{a_{2}}\left(1+\tilde{a_{1}}\right)}{\mathcal{Q}^{*} \Gamma\left(\kappa^{*}\right)}\right| \mid\left(\int_{1}^{e}\left(\ln \frac{e}{\varpi}\right)^{\kappa^{*}-1}\left[\hat{\Upsilon}(\varpi, y(\varpi))-\hat{\Upsilon}\left(\varpi, y^{\prime}(\varpi)\right) \frac{\mathrm{d} \varpi}{\varpi}\right] \mid\right. \\
& \quad+\left|\frac{\left(1+\tilde{a_{1}}\right)}{\mathcal{Q}^{*} \Gamma\left(\kappa^{*}+\theta^{*}-1\right)}\right| \mid\left(\int_{1}^{e}\left(\ln \frac{e}{\varpi}\right)^{\kappa^{*}+\theta^{*}-2}\left[\hat{\Upsilon}(\varpi, y(\varpi)) \frac{\mathrm{d} \varpi}{\varpi}-\hat{\Upsilon}\left(\varpi, y^{\prime}(\varpi)\right)\right] \mid\right. \\
& \leq L_{\hat{\Upsilon}}\left(\left|\frac{\tilde{a_{2}}\left(1+\tilde{a_{1}}\right)}{\mathcal{Q}^{*} \Gamma\left(\kappa^{*}+1\right)}\right|+\left|\frac{\left(1+\tilde{a_{1}}\right)}{\mathcal{Q}^{*} \Gamma\left(\kappa^{*}+\theta^{*}\right)}\right|\right)\left\|y-y^{\prime}\right\|_{\mathcal{X}^{\prime}}
\end{aligned}
$$

where $\Phi_{2}: \mathcal{X} \rightarrow \mathcal{X}$ is defined in (11). From (12) and (13), we have

$$
|\Phi(y)| \leq L_{\hat{\Upsilon}}\left(\frac{1}{\Gamma\left(\kappa^{*}+1\right)}+\left|\frac{\tilde{a_{2}}\left(1+\tilde{a_{1}}\right)}{\mathcal{Q}^{*} \Gamma\left(\kappa^{*}+1\right)}\right|+\left|\frac{\left(1+\tilde{a_{1}}\right)}{\mathcal{Q}^{*} \Gamma\left(\kappa^{*}+\theta^{*}\right)}\right|\right)\left\|y-y^{\prime}\right\|_{\mathcal{X}},
$$

which yields that $\Phi=\Phi_{1}+\Phi_{2}: \mathcal{X} \rightarrow \mathcal{X}$ is a contraction. By utilizing the Banach contraction principle, it is deduced that the mixed Caputo-Hadamard nonhybrid BVP (3) has a unique solution.

\section{Examples}

In this part of the paper, we examine our theoretical results by presenting some numerical examples to show the applicability of the analytical findings.

Example 1 To illustrater the mixed Caputo-Hadamard hybrid BVP (1)-(2), we formulate the following hybrid equation:

$$
\begin{aligned}
& { }^{\mathcal{C H}} \mathcal{D}_{1^{+}}^{1.78}\left[\frac{y(t)-\frac{1}{2+t}\left(y(t)+\cos \left(-\frac{1}{9} \int_{1}^{e} y(\varpi) \mathrm{d} \varpi\right)+\sin \left(\mathcal{H}_{1^{+}}^{0.33} y(t)\right)\right)+0.2021}{\frac{t}{2020}\left(y(t)+\frac{\frac{1}{27} \int_{1}^{e} y(\varpi) \mathrm{d} \varpi+\mathcal{H}^{2} \mathcal{I}_{1^{2}}^{2.1} y(t)}{1+\frac{1}{27} \int_{1}^{e} y(\varpi) \mathrm{d} \varpi+\mathcal{I}_{1^{+}}^{2.11} y(t)}\right)+0.11}\right] \\
& =(1+t)^{2} \sin (y(t))
\end{aligned}
$$


furnished with mixed Hadamard integral hybrid boundary conditions

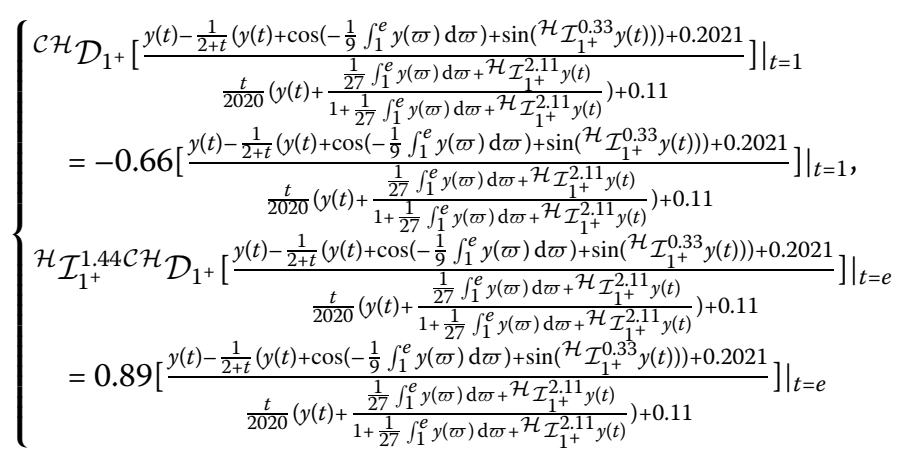

so that $t \in[1, e], \kappa^{*}=1.78, \gamma^{*}=0.33, \mu^{*}=2.11, \theta^{*}=1.44, \lambda_{1}^{*}=\frac{-1}{9}, \lambda_{2}^{*}=\frac{1}{27}, \tilde{a_{1}}=-0.66$, and $\tilde{a}_{2}=0.89$. Define the function $\hat{\Upsilon}:[1, e] \times \mathbb{R} \rightarrow \mathbb{R}$ by $\hat{\Upsilon}(t, y(t))=(1+t)^{2} \sin (y(t))$. Obviously, $\hat{\Upsilon} \in \mathcal{C}_{\mathbb{R}}([1, e] \times \mathbb{R})$. Now, put $\psi(t)=(1+t)^{2}$ and $\xi(\|y\|)=1$. Thus $\psi^{*} \approx 13.8256$. Further, define two continuous maps $\Lambda:[1, e] \times \mathbb{R}^{3} \rightarrow \mathbb{R}$ and $\Psi:[1, e] \times \mathbb{R}^{3} \rightarrow \mathbb{R} \backslash\{0\}$ by

$$
\begin{aligned}
& \Lambda\left(t, y(t),-\frac{1}{9} \int_{1}^{e} y(\varpi) \mathrm{d} \varpi,{ }^{\mathcal{H}} \mathcal{I}_{1^{+}}^{0.33} y(t)\right) \\
& \quad=\frac{1}{2+t}\left(y(t)+\cos \left(-\frac{1}{9} \int_{1}^{e} y(\varpi) \mathrm{d} \varpi\right)+\sin \left({ }^{\mathcal{H}} \mathcal{I}_{1^{+}}^{0.33} y(t)\right)\right)+0.2021
\end{aligned}
$$

and

$$
\begin{aligned}
& \Psi\left(t, y(t), \lambda_{2}^{*} \int_{1}^{e} y(\varpi) \mathrm{d} \varpi,{ }^{\mathcal{H}} \mathcal{I}_{1^{+}}^{\mu^{*}} y(t)\right) \\
& =\frac{t}{2020}\left(y(t)+\frac{\frac{1}{27} \int_{1}^{e} y(\varpi) \mathrm{d} \varpi+{ }^{\mathcal{H}} \mathcal{I}_{1^{+}}^{2.11} y(t)}{1+\frac{1}{27} \int_{1}^{e} y(\varpi) \mathrm{d} \varpi+{ }^{\mathcal{H}} \mathcal{I}_{1^{+}}^{2.11} y(t)}\right)+0.11 .
\end{aligned}
$$

Note that $\Lambda^{*} \approx 0.2021$ and $\Psi^{*}=0.11$. We claim that function $\Lambda$ is Lipschitz. To see this, for every $y, y^{\prime} \in \mathbb{R}$, we have

$$
\begin{aligned}
& \mid \Lambda\left(t, y_{1}(t),-\frac{1}{9} \int_{1}^{e} y_{1}(\varpi) \mathrm{d} \varpi,{ }^{\mathcal{H}} \mathcal{I}_{1^{+}}^{0.33} y_{1}(t)\right) \\
& \quad-\Lambda\left(t, y_{2}(t),-\frac{1}{9} \int_{1}^{e} y_{2}(\varpi) \mathrm{d} \varpi,{ }^{\mathcal{H}} \mathcal{I}_{1^{+}}^{0.33} y_{2}(t)\right) \mid \\
& \leq \frac{1}{2+t}\left[1+\left|\frac{-1}{9}(e-1)\right|+\frac{1}{\Gamma(0.33+1)}\right] \sup _{t \in[1, e]}\left|y_{1}(t)-y_{2}(t)\right| .
\end{aligned}
$$

Letting $\sigma(t)=\frac{1}{2+t}$, we have $\sigma^{*}\left[1+\left|\frac{-1}{9}(e-1)\right|+\frac{1}{\Gamma(0.33+1)}\right] \approx 0.8500$. In a similar manner, function $\Psi$ is also Lipschitz. Indeed, for every $y, y^{\prime} \in \mathbb{R}$, we have

$$
\begin{aligned}
& \mid \Psi\left(t, y_{1}(t), \frac{1}{27} \int_{1}^{e} y_{1}(\varpi) \mathrm{d} \varpi,{ }^{\mathcal{H}} \mathcal{I}_{1^{+}}^{2.11} y_{1}(t)\right) \\
& \quad-\Psi\left(t, y_{2}(t), \frac{1}{27} \int_{1}^{e} y_{2}(\varpi) \mathrm{d} \varpi,{ }^{\mathcal{H}} \mathcal{I}_{1^{+}}^{2.11} y_{2}(t)\right) \mid
\end{aligned}
$$




$$
\begin{aligned}
& \leq \frac{t}{2020}\left[1+\left|\frac{1}{27}(e-1)\right|+\frac{1}{\Gamma(2.11+1)}\right] \sup _{t \in[1, e]}\left|y_{1}(t)-y_{2}(t)\right| \\
& \leq \varrho(t)\left[1+\left|\lambda_{2}^{*}(e-1)\right|+\frac{1}{\Gamma\left(\mu^{*}+1\right)}\right] \sup _{t \in[1, e]}\left|y_{1}(t)-y_{2}(t)\right|
\end{aligned}
$$

so that $\varrho^{*}\left[1+\left|\lambda_{2}^{*}(e-1)\right|+\frac{1}{\Gamma\left(\mu^{*}+1\right)}\right]=\frac{e}{2020}\left[1+\left|\frac{1}{27}(e-1)\right|+\frac{1}{\Gamma(2.11+1)}\right]=0.0020$. Eventually, we obtain $\tilde{M} \approx 0.9980$ and select $\rho>0.8975>0$. In addition,

$$
\begin{aligned}
& \varrho^{*}\left[1+\left|\lambda_{2}^{*}(e-1)\right|+\frac{1}{\Gamma\left(\mu^{*}+1\right)}\right] \psi^{*} \xi(\|y\| \mathcal{X}) \tilde{M}+\sigma^{*}\left[1+\left|\lambda_{1}^{*}(e-1)\right|+\frac{1}{\Gamma\left(\gamma^{*}+1\right)}\right] \\
& \quad \approx 0.6057<1 .
\end{aligned}
$$

Hence, by invoking Theorem 8 , it is realized that the mixed Caputo-Hadamard hybrid BVP (14)-(15) has a solution on $[1, e]$.

Example 2 To illustrate the mixed Caputo-Hadamard nonhybrid BVP (3), we formulate the following nonhybrid BVP:

$$
\left\{\begin{array}{l}
{ }^{\mathrm{CH}} \mathcal{D}_{1^{+}}^{1.14} y(t)=\frac{1}{49+\exp \left(t^{2}-1\right)} \frac{|y(t)|}{(1+|y(t)|)}, \\
{ }^{{ }^{C H}} \mathcal{D}_{1^{+}} y(1)=-1.66 y(1), \quad \frac{1}{\Gamma(0.74)} \int\left(\ln \frac{e}{\sigma}\right)^{-0.26}\left[{ }^{C H} \mathcal{D}_{1^{+}} y(\varpi)\right] \frac{\mathrm{d} \sigma}{\varpi}=0.56 y(e),
\end{array}\right.
$$

so that $t \in[1, e], \kappa^{*}=1.14, \theta^{*}=0.74, \tilde{a_{1}}=-1.66$, and $\tilde{a_{2}}=0.56$. Then, an integral structure of the solution for the mixed Caputo-Hadamard nonhybrid BVP (16) is represented by

$$
\begin{aligned}
y(t)= & \frac{1}{\Gamma(1.14)} \int_{1}^{t}\left(\ln \frac{t}{\varpi}\right)^{0.14} \hat{\Upsilon}(t, y(t)) \frac{\mathrm{d} \varpi}{\varpi} \\
& +\frac{0.56(1-1.66 \ln (t))}{\mathcal{Q}^{*} \Gamma(1.14)} \int_{1}^{e}\left(\ln \frac{e}{\varpi}\right)^{0.14} \hat{\Upsilon}(t, y(t)) \frac{\mathrm{d} \varpi}{\varpi} \\
& -\frac{(1-1.66 \ln (t))}{\mathcal{Q}^{*} \Gamma(0.88)} \int_{1}^{e}\left(\ln \frac{e}{\varpi}\right)^{-0.12} \hat{\Upsilon}(t, y(t)) \frac{\mathrm{d} \varpi}{\varpi}
\end{aligned}
$$

for any $t \in[1, e]$, where the continuous function $\hat{\Upsilon}:[1, e] \times \mathbb{R} \rightarrow \mathbb{R}$ is defined by

$$
\hat{\Upsilon}(t, y(t))=\frac{1}{49+\exp \left(t^{2}-1\right)}\left(\frac{|y(t)|}{1+|y(t)|}\right) .
$$

Then, one can write

$$
\left|\hat{\Upsilon}(t, y(t))-\hat{\Upsilon}\left(t, y^{\prime}(t)\right)\right| \leq \frac{1}{50}\left\|y(t)-y^{\prime}(t)\right\|_{\mathbb{R}}
$$

and

$$
|\hat{\Upsilon}(t, y(t))| \leq \frac{1}{50}|y(t)|
$$

with $L_{\hat{\Upsilon}}=\frac{1}{50}, C_{\hat{\Upsilon}}=\frac{1}{50}$, and $M_{\hat{\Upsilon}}=0$. Now, define three operators $\Phi_{1}, \Phi_{2}, \Phi: \mathbb{R} \rightarrow \mathbb{R}$ as follows:

$$
\left(\Phi_{1} y\right)(t)=\frac{1}{\Gamma(1.14)} \int_{1}^{t}\left(\ln \frac{t}{\varpi}\right)^{0.14} \hat{\Upsilon}(t, y(t)) \frac{\mathrm{d} \varpi}{\varpi},
$$




$$
\begin{aligned}
\left(\Phi_{2} y\right)(t)= & \frac{0.56(1-1.66 \ln (t))}{\mathcal{Q}^{*} \Gamma(1.14)} \int_{1}^{e}\left(\ln \frac{e}{\varpi}\right)^{0.14} \hat{\Upsilon}(t, y(t)) \frac{\mathrm{d} \varpi}{\varpi} \\
& -\frac{(1-1.66 \ln (t))}{\mathcal{Q}^{*} \Gamma(0.88)} \int_{1}^{e}\left(\ln \frac{e}{\varpi}\right)^{-0.12} \hat{\Upsilon}(t, y(t)) \frac{\mathrm{d} \varpi}{\varpi}
\end{aligned}
$$

and $(\Phi y)(t)=\left(\Phi_{1} y\right)(t)+\left(\Phi_{2} y\right)(t)$. Since $\Phi_{1}$ and $\Phi_{2}$ are continuous and bounded, $\Phi=\Phi_{1}+$ $\Phi_{2}$ is continuous and bounded, too. Further, we have

$$
\left|\Phi_{1} y(t)-\Phi_{1} y^{\prime}(t)\right| \leq \frac{1}{50 \times \Gamma(2.14)}\left\|y-y^{\prime}\right\|_{\mathbb{R}}
$$

which implies that $\Phi_{1}$ is $\mu$-Lipschitz with constant $\tilde{K}_{1}^{*}=\frac{1}{50 \times \Gamma(2.14)}$ by Proposition 2 . Also, by using hypothesis $(\mathcal{H P} 6)$ for the growth condition, we get $\left\|\Phi_{1}(y)\right\|_{\mathbb{R}} \leq \frac{1}{50 \times \Gamma(2.14)}\|y\|_{\mathbb{R}}$. Therefore, since $\Phi_{1}$ is $\mu$-Lipschitz with constant $\tilde{K}_{1}^{*}=\frac{1}{50 \times \Gamma(2.14)}$ and $\Phi_{2}$ is compact with constant $\tilde{K}_{2}^{*}=0$, by Proposition $4, \Phi=\Phi_{1}+\Phi_{2}$ is a strict $\mu$-contraction with constant $\tilde{K}^{*}=\tilde{K}_{1}^{*}+\tilde{K}_{2}^{*}=\frac{1}{50 \times \Gamma(2.14)} \simeq 0.0187<1$. Thus $\Phi$ is a $\mu$-condensing operator. Also, take

$$
\mathcal{B}=\left\{y \in C_{\mathbb{R}}([1, e]): \text { there is } \lambda \in[0,1] \text { such that } y=\frac{1}{2}(\Phi y)\right\} \text {. }
$$

Then $\|y\|_{\mathbb{R}} \leq \frac{1}{2}\|\Phi y\|_{\mathbb{R}} \leq 1$ implies that $\mathcal{B}$ is a bounded set and so, by Theorem 13, it is deduced that the mixed Caputo-Hadamard nonhybrid BVP (16) has at least one solution $y$ in $C_{\mathbb{R}}([1, e])$. In addition,

$$
L_{\hat{\Upsilon}}\left(\frac{1}{\Gamma\left(\kappa^{*}+1\right)}+\left|\frac{\tilde{a_{2}}\left(1+\tilde{a_{1}}\right)}{\mathcal{Q}^{*} \Gamma\left(\kappa^{*}+1\right)}\right|+\left|\frac{\left(1+\tilde{a_{1}}\right)}{\mathcal{Q}^{*} \Gamma\left(\kappa^{*}+\theta^{*}\right)}\right|\right) \approx 0.0331<1 .
$$

Therefore, Theorem 14 implies that the mixed Caputo-Hadamard nonhybrid BVP (16) has a unique solution.

\section{Conclusion}

The fractional calculus has always been one of the most widely used branches of mathematics in other applied and computational sciences. This degree of importance is due to the high flexibility of the tools and operators defined in this theory. On this basis, researchers have been using various powerful fractional operators in recent decades to model different types of existing natural processes in the world. In the current research article, two hybrid and nonhybrid fractional BVPs of Caputo-Hadamard type are addressed. We seek the existence criteria for these two problems separately. We first utilize the generalized Dhage's theorem to derive desired results for an integral structure of solutions for the proposed hybrid BVP (1)-(2). Next, we establish other results for nonhybrid BVP (3) based on some existing notions in the topological degree theory. At the end of the paper, we examine our theoretical results by presenting some numerical examples to show the applicability of the analytical findings. 
Funding

Not applicable.

\section{Availability of data and materials}

Data sharing not applicable to this article as no datasets were generated or analyzed during the current study.

Ethics approval and consent to participate

Not applicable.

\section{Competing interests}

The authors declare that they have no competing interests.

\section{Consent for publication}

Not applicable.

\section{Authors' contributions}

The authors declare that the study was realized in collaboration with equal responsibility. All authors read and approved the final manuscript.

\section{Author details}

'Laboratory of Applied Mathematics, University of Kasdi Merbah, Ouargla 30000, Algeria. ${ }^{2}$ Department of Mathematics, Azarbaijan Shahid Madani University, Tabriz, Iran. ${ }^{3}$ Institute of Research and Development, Duy Tan University, Da Nang 550000, Vietnam. ${ }^{4}$ Faculty of Natural Sciences, Duy Tan University, Da Nang 550000, Vietnam. ${ }^{5}$ Department of Medical Research, China Medical University Hospital, China Medical University, Taichung, Taiwan.

\section{Publisher's Note}

Springer Nature remains neutral with regard to jurisdictional claims in published maps and institutional affiliations.

Received: 18 May 2020 Accepted: 13 July 2020 Published online: 18 July 2020

\section{References}

1. Alizadeh, S., Baleanu, D., Rezapour, S.: Analyzing transient response of the parallel RCL circuit by using the Caputo-Fabrizio fractional derivative. Adv. Differ. Equ. 2020, 55 (2020). https://doi.org/10.1186/s13662-020-2527-0

2. Baleanu, D., Jajarmi, A., Mohammadi, H., Rezapour, S.: Analysis of the human liver model with Caputo-Fabrizio fractional derivative. Chaos Solitons Fractals 134, 7 (2020)

3. Baleanu, D., Mohammadi, H., Rezapour, S.: Analysis of the model of HIV-1 infection of CD4+ T-cell with a new approach of fractional derivative. Adv. Differ. Equ. 2020, 71 (2020) https://doi.org/10.1186/s13662-020-02544-w

4. Baleanu, D., Mohammadi, H., Rezapour, S.: A mathematical theoretical study of a particular system of Caputo-Fabrizio fractional differential equations for the rubella disease model. Adv. Differ. Equ. 2020, 184 (2020). https://doi.org/10.1186/s13662-020-02614-z

5. Wang, G., Ren, X.: Radial symmetry of standing waves for nonlinear fractional Laplacian Hardy-Schrödinger systems. Appl. Math. Lett. 110, 106560 (2020). https://doi.org/10.1016/j.aml.2020.106560

6. Wang, G., Qin, J., Zhang, L., Baleanu, D.: Explicit iteration to a nonlinear fractional Langevin equation with non-separated integro-differential strip-multi-point boundary conditions. Chaos Solitons Fractals 131, 109476 (2020) https://doi.org/10.1016/j.chaos.2019.109476

7. Wang, G., Hou, W.: Standing waves of nonlinear fractional $p$-Laplacian Schrödinger equation involving logarithmic nonlinearity. Appl. Math. Lett. 102, 106149 (2020). https://doi.org/10.1016/j.aml.2019.106149

8. Wang, G., Ren, X., Bai, Z., Hou, W.: Radial symmetry of standing waves for nonlinear fractional Hardy-Schrodinger equation. Appl. Math. Lett. 96, 131-137 (2019). https://doi.org/10.1016/j.aml.2019.04.024

9. Wang, G., Pei, K., Agarwal, R.P., Zhang, L., Ahmad, B.: Nonlocal Hadamard fractional boundary value problem with Hadamard integral and discrete boundary conditions on a half-line. J. Comput. Appl. Math. 343, 230-239 (2018). https://doi.org/10.1016/j.cam.2018.04.062

10. Pei, K., Wang, G., Sun, Y.: Successive iterations and positive extremal solutions for a Hadamard type fractional integro-differential equations on infinite domain. Appl. Math. Comput. 312, 158-168 (2017). https://doi.org/10.1016/j.amc.2017.05.056

11. Baleanu, D., Mohammadi, H., Rezapour, S.: A fractional differential equation model for the COVID-19 transmission by using the Caputo-Fabrizio derivative. Adv. Differ. Equ. 2020, 299 (2020). https://doi.org/10.1186/s13662-020-02762-2

12. Charandabi, Z.Z., Rezapour, S., Ettefagh, M.: On a fractional hybrid version of the Sturm-Liouville equation. Adv. Differ. Equ. 2020, 301 (2020). https://doi.org/10.1186/s13662-020-02765-z

13. Etemad, S., Rezapour, S., Samei, M.E.: $\alpha-\psi$-contractions and solutions of a q-fractional differential inclusion with three-point boundary value conditions via computational results. Adv. Differ. Equ. 2020, 218 (2020). https://doi.org/10.1186/s13662-020-02679-w

14. Etemad, S., Rezapour, S., Sakar, F.M.: On a fractional Caputo-Hadamard problem with boundary value conditions via different orders of the Hadamard fractional operators. Adv. Differ. Equ. 2020, 272 (2020). https://doi.org/10.1186/s13662-020-02741-7

15. Etemad, S., Rezapour, S.: On the existence of solutions for fractional boundary value problems on the Ethane graph. Adv. Differ. Equ. 2020, 276 (2020). https://doi.org/10.1186/s13662-020-02736-4

16. Baleanu, D., Etemad, S., Rezapour, S.: A hybrid Caputo fractional modeling for thermostat with hybrid boundary value conditions. Bound. Value Probl. 2020, 64 (2020). https://doi.org/10.1186/s13661-020-01361-0

17. Baleanu, D., Ghafarnezhad, K., Rezapour, S., Shabibi, M.: On the existence of solutions of a three steps crisis integro-differential equation. Adv. Differ. Equ. 2018, 135 (2018) 
18. Baleanu, D., Ghafarnezhad, K., Rezapour, S.: On a three steps crisis integro-differential equation. Adv. Differ. Equ. 2019, 153 (2019)

19. Akbari Kojabad, E., Rezapour, S.: Approximate solutions of a sum-type fractional integro-differential equation by using Chebyshev and Legendre polynomials. Adv. Differ. Equ. 2017, 351 (2017)

20. Aydogan, S.M., Baleanu, D., Mousalou, A., Rezapour, S.: On high order fractional integro-differential equations including the Caputo-Fabrizio derivative. Bound. Value Probl. 2018, 90 (2018)

21. Aydogan, S.M., Baleanu, D., Mousalou, A., Rezapour, S.: On approximate solutions for two higher-order Caputo-Fabrizio fractional integro-differential equations. Adv. Differ. Equ. 2017, 251 (2017)

22. Baleanu, D., Rezapour, S., Mohammadi, H.: Some existence results on nonlinear fractional differential equations. Philos. Trans. R. Soc. A 371, 20120144 (2013). https://doi.org/10.1098/rsta.2012.0144

23. Baleanu, D., Mousalou, A., Rezapour, S.: A new method for investigating approximate solutions of some fractional integro-differential equations involving the Caputo-Fabrizio derivative. Adv. Differ. Equ. 2017(1), 51 (2017). https://doi.org/10.1186/s13662-017-1088-3

24. Baleanu, D., Mousalou, A., Rezapour, S.: The extended fractional Caputo-Fabrizio derivative of order $0 \leq \sigma<1$ on $C_{\mathbb{R}}[0,1]$ and the existence of solutions for two higher-order series-type differential equations. Adv. Differ. Equ. 2018(1), 255 (2018). https://doi.org/10.1186/s13662-018-1696-6

25. Baleanu, D., Mousalou, A., Rezapour, S.: On the existence of solutions for some infinite coefficient-symmetric Caputo-Fabrizio fractional integro-differential equations. Bound. Value Probl. 2017(1), 145 (2017). https://doi.org/10.1186/s13661-017-0867-9

26. Etemad, S., Pourrazi, S., Rezapour, S.: On a hybrid inclusion problem via hybrid boundary value conditions. Adv. Differ. Equ. 2020, 302 (2020). https://doi.org/10.1186/s13662-020-02764-0

27. Baleanu, D., Rezapour, S., Saberpour, Z:: On fractional integro-differential inclusions via the extended fractional Caputo-Fabrizio derivation. Bound. Value Probl. 2019, 79 (2019). https://doi.org/10.1186/s13661-019-1194-0

28. Talaee, M., Shabibi, M., Gilani, A., Rezapour, S.: On the existence of solutions for a pointwise defined multi-singular integro-differential equation with integral boundary condition. Adv. Differ. Equ. 2020, 41 (2020). https://doi.org/10.1186/s13662-020-2517-2

29. Ardjouni, A., Djoudi, A.: Positive solutions for nonlinear Caputo-Hadamard fractional differential equations with integral boundary conditions. Open J. Math. Anal. 3, 62-69 (2019)

30. Derbazi, C., Hammouche, H.: Caputo-Hadamard fractional differential equations with nonlocal fractional integro-differential boundary conditions via topological degree theory. AlMS Math. 5(3), 2694-2709 (2020)

31. Samei, M.E., Hedayati, V., Rezapour, S.: Existence results for a fractional hybrid differential inclusion with Caputo-Hadamard type fractional derivative. Adv. Differ. Equ. 2019, 163 (2019)

32. Tariboon, J., Cuntavepanit, A., Ntouyas, S.K., Nithiarayaphaks, W.: Separated boundary value problems of sequential Caputo and Hadamard fractional differential equations. J. Funct. Spaces Appl. 2018, 8 (2018). https://doi.org/10.1155/2018/6974046

33. Amara, A.: Existence results for hybrid fractional differential equations with three-point boundary conditions. AIMS Math. 5(2), 1074-1088 (2020)

34. Baleanu, D., Etemad, S., Pourrazi, S., Rezapour, S.: On the new fractional hybrid boundary value problems with three-point integral hybrid conditions. Adv. Differ. Equ. 2019, 473 (2019)

35. Baleanu, D., Hedayati, V., Rezapour, S., Al Qurashi, M.M.: On two fractional differential inclusions. SpringerPlus 5(1), 882 (2016). https://doi.org/10.1186/s40064-016-2564-z

36. Derbazi, C., Hammouche, H., Benchohra, M., Zhou, Y.: Fractional hybrid differential equations with three-point boundary hybrid conditions. Adv. Differ. Equ. 2019, 125 (2019)

37. Etemad, S., Rezapour, S., Samei, M.E.: On fractional hybrid and non-hybrid multi-term integro-differential inclusions with three-point integral hybrid boundary conditions. Adv. Differ. Equ. 2020, 161 (2020)

38. Hilal, K., Kajouni, A.: Boundary value problems for hybrid differential equations with fractional order. Adv. Differ. Equ. 2015, $183(2015)$

39. Sun, S., Zhao, Y., Han, Z., Li, Y.: The existence of solutions for boundary value problem of fractional hybrid differential equations. Commun. Nonlinear Sci. Numer. Simul. 17, 4961-4967 (2012)

40. Dhage, B.C., Lakshmikantham, V.: Basic results on hybrid differential equation. Nonlinear Anal. Hybrid Syst. 4, 414-424 (2010)

41. Zhao, Y., Sun, S., Han, Z., Li, Q.: Theory of fractional hybrid differential equations. Comput. Math. Appl. 62, 1312-1324 (2011)

42. Ullah, Z., Ali, A., Khan, R.A., Iqbal, M.: Existence results to a class of hybrid fractional differential equations. Matrix Sci. Math. 2(1), 13-17 (2018)

43. Ahmad, B., Nieto, J.J.: Existence of solutions for anti-periodic boundary value problems involving fractional differential equations via Leray-Schauder degree theory. Topol. Methods Nonlinear Anal. 35, 295-304 (2010)

44. Nanware, A., Dhaigude, D.B.: Existence and uniqueness of solutions of differential equations of fractional order with integral boundary conditions. J. Nonlinear Sci. Appl. 7, 246-254 (2014)

45. Shah, K., Ali, A., Khan, R.A.: Degree theory and existence of positive solutions to coupled systems of multi-point boundary value problems. Bound. Value Probl. 2016, 43 (2016)

46. Shah, K., Hussain, W.: Investigating a class of nonlinear fractional differential equations and its Hyers-Ulam stability by means of topological degree theory. Numer. Funct. Anal. Optim. 49, 1355-1372 (2019)

47. Wang, J., Zhou, Y., Wei, W.: Study in fractional differential equations by means of topological degree methods. Numer. Funct. Anal. Optim. 33(2), 216-238 (2012)

48. Zada, M.B., Shah, K., Khan, R.A.: Existence theory to a coupled system of higher order fractional hybrid differential equations by topological degree theory. Int. J. Appl. Comput. Math. 4, 102 (2018). https://doi.org/10.1007/s40819-018-0534-6

49. Aljoudi, S., Ahmad, B., Nieto, J.J., Alsaedi, A.: A coupled system of Hadamard type sequential fractional differential equations with coupled strip conditions. Chaos Solitons Fractals 91, 39-46 (2016). https://doi.org/10.1016/j.chaos.2016.05.005

50. Kilbas, A.A., Srivastava, H.M., Trujillo, J.J.: Theory and Applications of Fractional Differential Equations. Elsevier, Amsterdam (2006) 
51. Agarwal, R.P., O’Regan, D.: Topological Degree Theory and Its Applications. Taylor \& Francis, New York (2006)

52. Deimling, K.: Multi-Valued Differential Equations. de Gruyter, Berlin (1992)

53. Isaia, F.: On a nonlinear integral equation without compactness. Acta Math. Univ. Comen. 75, 233-240 (2006)

54. Dhage, B.C.: A fixed point theorem in Banach algebras with applications to functional integral equations. Kyungpook Math. J. 44, 145-155 (2004)

Submit your manuscript to a SpringerOpen ${ }^{\odot}$ journal and benefit from:

- Convenient online submission

$\checkmark$ Rigorous peer review

Open access: articles freely available online

- High visibility within the field

- Retaining the copyright to your article

Submit your next manuscript at $\gg$ springeropen.com 